Les lasers et leurs applications scientifiques et médicales

\title{
Laser monofréquence et stabilisation
}

\author{
Ch. Chardonnet
}

Laboratoire de Physique des Lasers, URA 282 du CNRS, Université Paris XIII, Avenue J.-B. Clément, 93430 Villetaneuse, France

\section{INTRODUCTION}

La lumière émise par un laser continu et monomode possède d'emblée une largeur spectrale extrêmement étroite. Si l'on considère un laser à colorant de fréquence $v=600 \mathrm{THz}$, une largeur de raie de $\delta \mathrm{v}=10 \mathrm{MHz}$ correspond à un coefficient de surtension $\mathrm{Q}=\mathrm{v} / \delta \mathrm{v}=6.10^{7}$. Néanmoins, dans de nombreuses applications, les performances de l'oscillateur doivent être bien supérieures à cela. La stabilité de la phase ou de la fréquence de l'oscillateur doit être assurée sur des échelles de temps plus ou moins longues. En outre, un laser dit "libre" émet à une fréquence moyenne imposée par la longueur optique de la cavité qui est susceptible de dériver de façon incontrôlée au cours du temps. Enfin, dans nombre de cas, il est nécessaire de pouvoir faire varier de façon très précise la fréquence du laser au cours du temps.

Si nous considérons le champ laser sous la forme suivante :

$$
E=E_{0} \exp \left(i \omega_{0} t+\varphi(t)\right)
$$

la fréquence instantanée du laser est reliée à la phase par la relation :

$$
v(t)=v_{0}+\dot{\varphi}(t) / 2 \pi
$$

où $v_{0}=\omega_{0} / 2 \pi$ représente la fréquence moyenne du laser. L'expression (1) du champ laser suppose une amplitude constante. En fait, les fluctuations d'amplitude contribuent elles-mêmes à la largeur spectrale du laser. Néanmoins, ces fluctuations peuvent être corrigées indépendamment des fluctuations de phase, en stabilisant l'intensité lumineuse. Dans ce cas, les fluctuations peuvent être ramenées au niveau du bruit quantique de la lumière ou shot-noise (voire en dessous, par des méthodes de squeezing de la lumière). La largeur de raie laser est alors surtout déterminée par les fluctuations de phase.

L'amélioration des propriétés spectrales d'une fréquence laser ainsi que le contrôle de sa valeur au cours du temps sont obtenues par l'utilisation de méthodes de stabilisation. La qualité de la stabilisation en fréquence d'un laser dépend de trois paramètres :

- la référence de fréquence à laquelle la fréquence laser doit être comparée. Il peut s'agir de la fréquence d'un laser beaucoup plus stable, de la fréquence de résonance d'une cavité FabryPerot, d'une fréquence de transition atomique ou moléculaire.

- le rapport signal-sur-bruit $(\mathrm{S} / \mathrm{B})$ du discriminateur de phase/fréquence nécessaire à l'élaboration d'un signal de correction. 
- la fonction de transfert de la ou des boucle(s) d'asservissement qui doivent être notamment adaptées aux temps de réponse des transducteurs qui permettent la correction de la phase ou de la fréquence du laser.

Le choix du système de stabilisation dépend fortement des performances de l'oscillateur recherchées pour l'application envisagée. C'est pourquoi dans un premier temps, nous donnerons quelques grandes classes d'applications des lasers stabilisés en fréquence. Ensuite, nous rappellerons les grandeurs physiques adaptées à l'étude des propriétés spectrales d'un oscillateur et les méthodes pour y accéder. Dans une troisième partie, nous décrirons les éléments-clés d'un asservissement : les références de fréquence, les discriminateurs de phase/fréquence qu'elles permettent d'élaborer ainsi que la limite ultime de largeur de raie qui peut ainsi être atteinte, et enfin, les limitations imposées par la boucle d'asservissement. Enfin, la dernière partie présentera des développements récents en matière de métrologie des fréquences optiques qui exploitent différentes méthodes de stabilisation.

\section{DES LASERS STABILISÉS, POUR QUOI FAIRE ?}

\subsection{Vélocimétrie}

On peut déterminer la vitesse d'un objet en mesurant le décalage Doppler que subit la lumière réfléchie par cet objet en mouvement.

Soit un objet dont on veut contrôler la vitesse avec une précision de $\delta \mathrm{v}=1 \mathrm{~cm} / \mathrm{s}$. L a fréquence de l'onde réfléchie est $v_{0}\left(1-\frac{2 v}{c}\right)$ où v représente la vitesse de l'objet suivant la normale au plan de réflexion. Pour résoudre $\delta \mathrm{v}$, il faut donc pouvoir résoudre un changement de fréquence de $\delta v=v_{0} \frac{2 \delta v}{\mathrm{c}}=\frac{2 \delta v}{\lambda_{0}}$. Si l'on utilise un laser à $\mathrm{CO}_{2}\left(\lambda_{0}=10 \mu \mathrm{m}\right)$, on devra mesurer un changement de $\delta v=2 \mathrm{kHz}$, soit un changement relatif de $\delta v / v=6 \cdot 10^{-11}$. Si cet objet est situé à $100 \mathrm{~km}$ de distance, le retard du signal réfléchi sera de $0.6 \mathrm{~ms}$. Autrement dit, une grande stabilité à court terme du laser sera recherchée pour ce type d'applications (mieux que $100 \mathrm{~Hz}\left(\delta \mathrm{v} / \mathrm{v}=3.10^{-12}\right)$ sur $0.1 \mathrm{~ms}$, typiquement). Par contre, une dérive à long terme de la fréquence moyenne du laser de l'ordre du $\mathrm{kHz} / \mathrm{s}$ n'affecte pas la résolution en vitesse. La stabilité à long terme n'est pas ici un critère essentiel.

Dans les projets d'expériences de détection d'ondes de gravitation, la situation est similaire puisque l'échelle de temps caractéristique de la détection pour les évènements les plus attendus est de l'ordre de la milliseconde, la sensibilité requise devra être de l'ordre de $\delta \mathrm{v} / \mathrm{v}=10^{-21}$.

\subsection{Métrologie des fréquences optiques}

La référence primaire de temps/fréquence est donnée par la fréquence micro-onde de l'atome de Césium au repos et isolé, à $9 \mathrm{GHz}$. Cette fréquence est une grandeur universelle, du moins si l'on suppose que les constantes fondamentales, e, c, $\hbar, \ldots$ ne varient pas au cours du temps. La réalisation de cette fréquence est, quant à elle, sujette à de nombreuses erreurs. On peut distinguer deux grandes classes d'erreurs :

- les erreurs dites statistiques incluent toutes les fluctuations de fréquences et dépendent de la nature des bruits de fréquences en présence dans le système. Nous verrons qu'en présence d'un bruit blanc de fréquence, la fréquence moyennée sur un temps $\tau$ de l'oscillateur 
$\langle v(t)\rangle_{\tau}=\frac{1}{\tau} \int_{t}^{t+\tau} v\left(t^{\prime}\right) d t^{\prime}$ tend vers une valeur fixe $v_{0}$ comme $\tau^{-1 / 2}$. C'est donc la stabilité à long terme qui constitue un paramètre essentiel de la qualité d'une référence de fréquence.

- la somme des erreurs systématiques représente l'écart entre cette fréquence moyenne $v_{0}$ et la fréquence de référence "vraie" correspondant à la définition de l'unité de temps. Les sources de ces erreurs peuvent être de nature instrumentale, par exemple une distorsion de la modulation nécessaire à l'élaboration du signal de référence et qui en affecte le centre. Ces sources d'erreurs peuvent être en principe réduites par des progrès instrumentaux. Il existe des sources d'erreurs liées au mode de réalisation de la référence de fréquence dont voici quelques exemples importants :

* l'effet Doppler du second ordre qui déplace la résonance atomique de $-\omega_{t B} \frac{v^{2}}{2 c^{2}}$ où v est le module de la vitesse de l'atome de césium,

* le déplacement par pression,

* le déplacement lumineux lié à la présence de l'onde en interaction avec les atomes,

* les champs électriques ou magnétiques extérieurs.

Ces erreurs peuvent être parfois évaluées et donc partiellement compensées. Expérimentalement, la précision ultime est une fraction de la largeur de la résonance qui est détectée. Une horloge à césium typique fournit un signal de frange de Ramsey de $\Delta v=100 \mathrm{~Hz}$ de largeur. Pour obtenir une précision relative de $\delta v / v=10^{-14}$, il faut déterminer le centre de la frange avec une précision de pointé de $\delta v / v=10^{-6}$. Ce niveau de performance n'a pu être atteint que par de nombreuses années d'effort sur les horloges à césium. Pour conserver le même niveau de précision relative sans disposer pour autant d'une telle précision du pointé, il faut soit augmenter le pouvoir de résolution de l'expérience (abaisser $\Delta v$ ) soit utiliser un standard de fréquence, $v$, plus élevée.
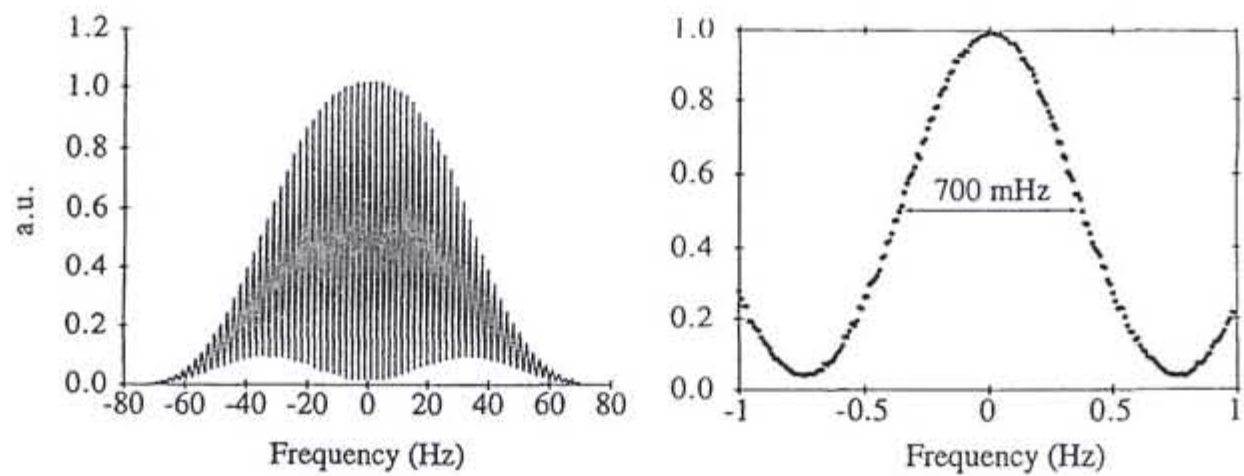

Figure 1: Exemple de signal de frange de Ramsey obtenu sur la transition du césium à $9192631770 \mathrm{~Hz}$, standard primaire de fréquence, sur une expérience de fontaine atomique [1] le progrès significatif sur la résolution par rapport aux horloges usuelles améliore sensiblement la stabilité à long terme.

La largeur d'une résonance, $\Delta v$, est proportionnelle à l'inverse du temps d'interaction cohérente de l'onde lumineuse avec les atomes. Cette largeur de $100 \mathrm{~Hz}$ est reliée au temps mis 
par les atomes de césium dans un jet thermique pour traverser les deux zones de champ distantes de plus d'un mètre. Un temps d'interaction d'une seconde $(\Delta v=1 \mathrm{~Hz})$ dans une expérience de fontaine atomique a pu être obtenu grâce à la possibilité de refroidissement des atomes par laser qui peuvent être stoppés et manipulés de façon contrôlée (Figure 1).

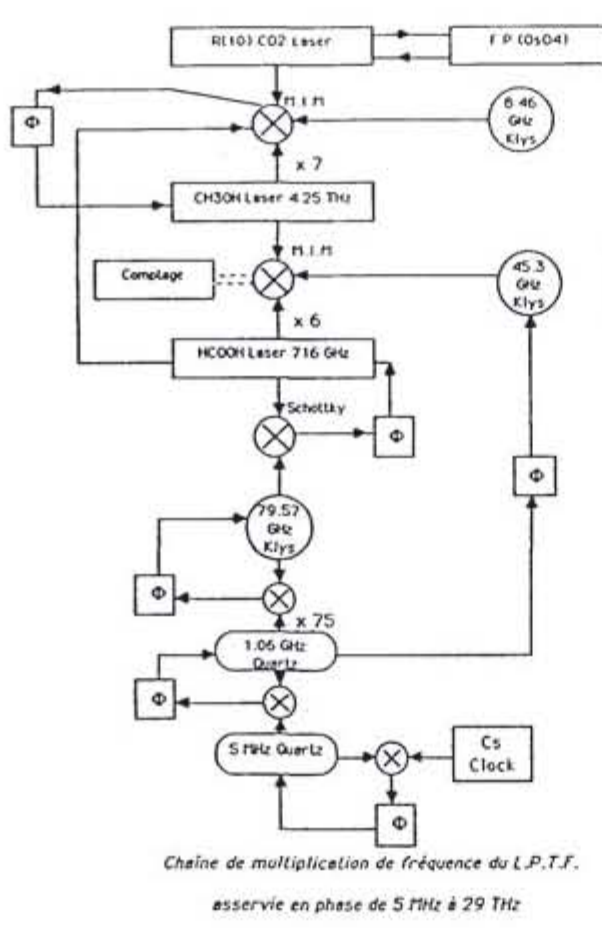

Figure 2 : chaîne de fréquences reliant l'horloge à césium vers $9 \mathrm{GHz}$ à la région spectrale de $10 \mu \mathrm{m}[2]$.
1992 LPTF liequency chain

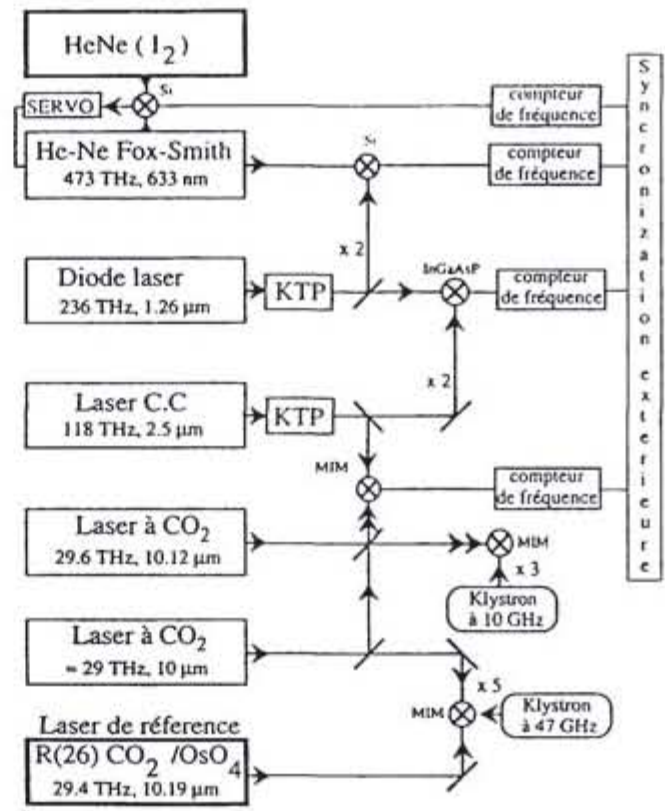

Figure 3 : chaîne de fréquences reliant la région spectrale de $10 \mu \mathrm{m}$ à la fréquence visible

[4]

L'autre voie consiste à utiliser une transition atomique ou moléculaire dans le domaine optique (infrarouge ou visible). Pour une résolution de $\Delta v=1 \mathrm{kHz}$, une précision de pointé de $6.10^{-3}$ est suffisante à $\mathrm{v}=600 \mathrm{THz}$ pour obtenir la même précision relative de $10^{-14}$. Expérimentalement, cela est plus facilement accessible. De telles fréquences de référence constituent des standards de fréquences secondaires dont les valeurs exactes ne peuvent être déterminées que par un raccordement au standard primaire délivré par une horloge à césium via un chaine de fréquences. Les Figures 2 et 3 montrent deux chaînes de fréquences, l'une (Figure 2) raccorde l'horloge à césium à la région spectrale de $10 \mu \mathrm{m}$ où des lasers à $\mathrm{CO}_{2}$ stabilisés sur des raies de $\mathrm{OsO}_{4}$ constituent des standards secondaires de fréquences. $\mathrm{La}$ seconde chaîne (Figure 3 ) utilise les standards secondaires de fréquences vers $10 \mu \mathrm{m}$ pour mesurer une fréquence visible. 


\subsection{Spectroscopie à haute résolution}

La spectroscopie laser nécessite une source de fréquence balayable continûment. Les spécifications de la source vont dépendre du niveau de résolution visé, de la précision de pointé escomptée et du temps d'intégration requis pour l'enregistrement des signaux. La largeur de raie laser doit être beaucoup plus faible que la résolution alors que la stabilité à long terme doit être grande devant la résolution pendant le temps d'accumulation du spectre.

Pour des expériences de faible résolution et pour laquelle une calibration en fréquence de l'ordre de la fraction de largeur de raie est suffisante, il est possible de s'affranchir des méthodes de stabilisation laser. On peut en effet balayer la fréquence du laser "libre" en appliquant une rampe de tension sur une céramique piézo-électrique portant un miroir de la cavité. L'enregistrement simultané au spectre des pics de transmission d'une cavité Fabry-Perot permet une calibration de l'axe des fréquences. Si une calibration absolue en fréquence est nécessaire, on peut enregistrer en parallèle un spectre moléculaire dense lui-même calibré (ex : Atlas de l'Iode).

Lorsque la résolution augmente, il devient rapidement indispensable d'utiliser des méthodes de stabilisation laser. Le principe de base adapté à la spectroscopie à très haute résolution consiste à utiliser deux lasers. Le premier laser est stabilisé sur une référence de fréquence très stable et est utilisé comme oscillateur local. On réalise alors le battement avec un second laser de fréquence "proche" et on asservit en phase ce battement sur une source radiofréquence ou micro-onde. Le balayage de cette dernière fréquence assure le balayage de la fréquence du second laser par rapport à l'oscillateur local. Si l'on admet que l'asservissement en phase permet de transférer pratiquement toutes les propriétés spectrales de l'oscillateur local sur le laser balayable, c'est donc la qualité de la référence de fréquence utilisée et les performances de l'asservissement de l'oscillateur local sur cette référence qui conditionnent les performances spectroscopiques ultimes (Figure 4).

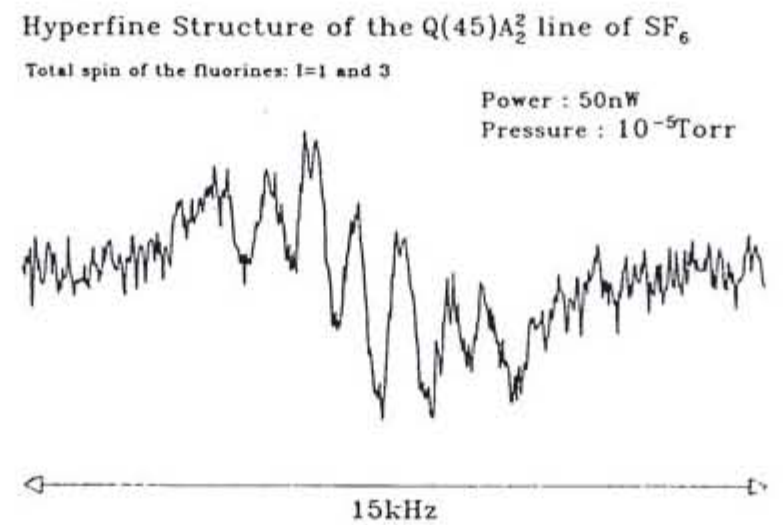

Figure 4 : Structure hyperfine d'une raie de $\mathrm{SF}_{6}$. Il s'agit de la structure la plus étroite résolue dans la région spectrale de $10 \mu \mathrm{m}$ ( 7 composantes sur $5.4 \mathrm{kHz}$ ). Une pureté spectrale de l'ordre de $10 \mathrm{~Hz}$ et une stabilité à long terme de l'ordre du $\mathrm{Hz} / \mathrm{mn}$ du laser à $\mathrm{CO}_{2}$ sont nécessaires pour atteindre cette résolution. [5]

\section{PROPRIÉTÉS SPECTRALES D'UN OSCILLATEUR}

Le champ laser n'est qu'un exemple d'oscillateur. Les définitions, théorèmes et propriétés utilisés couramment en théorie du signal sont, de ce fait, directement opérationnels pour caractériser les propriétés spectrales d'un champ laser. 


\subsection{Densité spectrale de puissance}

\subsubsection{Rappels}

$F(\omega)=\int_{-\infty}^{+\infty} f(t) \exp (-i \omega t) d t$ est la transformée de Fourier de $f(t)$.

$$
C_{f}(\tau)=\left\langle f(t+\tau) f^{*}(t)\right\rangle=\lim _{T \rightarrow+\infty} \frac{1}{2 T} \int_{-T}^{T} f(t+\tau) f^{*}(t) d t
$$

est la fonction d'autocorrélation de $f$.

Selon le théorème de Wiener-Khintchine, la densité spectrale de puissance associée à la fonction $f$ est la transformée de Fourier de la fonction d'autocorrélation :

$$
S_{f}(\omega)=\int_{-\infty}^{+\infty} C_{f}(\tau) \exp (-i \omega \tau) d \tau
$$

Alors la "puissance" totale associée à $f$ vérifie :

$$
P_{f}=C_{f}(0)=\int_{-\infty}^{+\infty} S_{f}(\omega) \frac{d \omega}{2 \pi}
$$

Par la suite, nous distinguerons les pulsations dans le domaine optique, $\omega$ et $\omega_{0}$, des pulsations plus basse fréquence, $\Omega$, ainsi que les fréquences optiques $v$ et $v_{0}$ des basses fréquences notées $f$.

3.1.2 relations entre les densités spectrales de puissance du champ, de fréquence, et de phase :

Soit $E(t)$ le champ laser considéré :

$$
E(t)=E_{0}(1+V(t)) \exp \left(i\left(\omega_{0} t+\varphi(t)\right)\right)
$$

$V(t)$ et $\varphi(t)$ représentent respectivement le bruit d'amplitude et le bruit de phase associés au champ laser et sont supposés varier lentement devant la pulsation optique $\omega_{0}$. Si nous négligeons la corrélation entre ces deux types de bruits, la fonction d'autocorrélation du champ électrique s'écrit :

$$
C_{E}(\tau)=E_{0}^{2} \exp \left(i \omega_{0} \tau\right)\left[1+C_{V}(\tau)\right]\langle\exp (i \varphi(t+\tau)-i \varphi(t))\rangle
$$

En utilisant le théorème des moments applicables à des processus gaussiens, on obtient :

$$
\langle\exp (i \varphi(t+\tau)-i \varphi(t))\rangle=\exp \left(-\frac{1}{2}\left\langle[\varphi(t+\tau)-\varphi(t)]^{2}\right\rangle\right)=\exp \left(C_{\varphi}(\tau)-C_{\varphi}(0)\right)
$$

et la puissance totale est :

$$
P_{E}=C_{E}(0)=E_{0}^{2}\left(1+C_{V}(0)\right)
$$

où nous voyons que seul le bruit d'amplitude ajoute une puissance supplémentaire à celle du champ électrique.

Si nous supposons que les bruits d'amplitude et de phase n'ont pas de cohérence à long terme, c'est à dire si :

$$
C_{V}(\tau)_{\tau \rightarrow \infty}=C_{\varphi}(\tau)_{\tau \rightarrow \infty}=0
$$

alors, nous avons : 


$$
C_{E}(\tau)_{\tau \rightarrow \infty}=E_{0}^{2} \exp \left(-C_{\varphi}(0)\right) \exp \left(i \omega_{0} \tau\right)=E_{0}^{2} \exp \left(-\varphi_{m s}^{2}\right) \exp \left(i \omega_{0} \tau\right)
$$

où $\varphi_{m s}^{2}$ représente la variance de la phase. L'équation (11) indique que la fraction de puissance restant dans la porteuse est réduite par le facteur $\exp \left(-\varphi_{m \mathrm{r}}^{2}\right)$. Par suite, si la phase diverge comme c'est le cas en présence d'un bruit blanc de fréquence, alors cette fraction de puissance est nulle.

Enfin, il est courant de considérer le bruit de fréquence plutôt que le bruit de phase. Si l'on considère qu'ils sont reliés par $f(t)=\frac{1}{2 \pi} \dot{\varphi}(t)$, les densités spectrales respectives vérifient la relation :

$$
S_{f}(\Omega)=\left(\frac{\Omega}{2 \pi}\right)^{2} S_{\varphi}(\Omega)
$$

\subsection{Largeur de raie laser}

La forme de raie laser est représentée par la densité spectrale de puissance associée au champ laser $E$ :

$$
S_{E}(\omega)=\int_{-\infty}^{+\infty} C_{E}(\tau) \exp (-i \omega \tau) d \tau
$$

où la fonction d'autocorrélation de E est obtenue à partir des équations (7) et (8) :

$$
C_{E}(\tau)=E_{0}^{2} \exp \left(i \omega_{0} \tau\right)\left[1+C_{V}(\tau)\right] \exp \left(C_{\varphi}(\tau)-C_{\varphi}(0)\right)
$$

Cette forme de raie est le signal qui serait obtenu avec un analyseur de spectre optique. En pratique, un analyseur de spectre dans le domaine radio-fréquence permettra d'observer une densité spectrale de puissance associée au battement entre deux lasers de fréquences voisines. Ce battement reproduira les propriétés spectrales du laser le moins stable ou le plus bruyant. Si les deux systèmes lasers sont identiques mais indépendants, les fluctuations de fréquence du battement résultent, à parts égales, de celles de chaque laser qui peut ainsi être caractérisé.

\subsubsection{Cas où le bruit de phase est faible}

Dans l'hypothèse d'un bruit de phase faible $\left(\left|C_{\varphi}(\tau)\right|<<1\right)$, cette expression peut être développée :

$$
C_{E}(\tau)=E_{0}^{2} \exp \left(-\varphi_{m s}^{2}\right)\left[1+C_{V}(\tau)+C_{\varphi}(\tau)\right] \exp \left(i \omega_{0} \tau\right)
$$

dont la transformée de Fourier (13) s'exprime aisément :

$$
S_{E}(\omega)=E_{0}^{2} \exp \left(-\varphi_{\text {rms }}^{2}\right)\left[\delta\left(\omega-\omega_{0}\right)+S_{V}\left(\omega-\omega_{0}\right)+S_{\varphi}\left(\omega-\omega_{0}\right)\right]
$$

La forme de raie est alors la somme de trois termes : le premier représente la porteuse à la fréquence $\omega_{0}$ dont l'amplitude est réduite par la présence du bruit de phase comme indiquée dans le §II.1.2. Les deux autres termes traduisent l'apparition de bandes latérales dues aux bruits de phase et d'amplitude. Ce spectre de bruit est simplement la somme des densités spectrales de puissance des bruits de phase et d'amplitude décalées de la fréquence égale à la porteuse.

Le cas d'un bruit blanc de phase est une application immédiate de la situation décrite cidessus. Expérimentalement, c'est le type de bruit obtenu lorsqu'on réalise un asservissement en 
phase idéal d'une fréquence laser sur un oscillateur de référence en présence (inévitable) du bruit quantique de la lumière ou shot noise. La densité spectrale de bruit de phase peut s'écrire dans ce cas :

$$
S_{\varphi}(\Omega)=\left\{\begin{array}{cc}
\frac{\varphi_{m s}^{2}}{2 B}, & |\Omega| \leq 2 \pi B \\
0, & |\Omega|>2 \pi B
\end{array}\right.
$$

La fonction d'autocorrélation associée au bruit de phase s'écrit :

$$
C_{\varphi}(\tau)=\varphi_{m s}^{2} \frac{\sin (2 \pi B \tau)}{2 \pi B \tau}
$$

La fonction prend des valeurs négligeables dès que $\tau \gg 1 / B$, temps au-delà duquel le système perd la "mémoire" de la phase initiale. La densité spectrale de puissance du champ laser est alors donnée avec une bonne approximation par l'équation (16) : il est constitué d'une porteuse à la fréquence laser $\omega_{0}$ et du bruit blanc centré à $\omega_{0}$ dont la largeur est la bande passante du bruit de phase. La puissance perdue de la porteuse se retrouve exactement dans ce bruit car une modulation de phase n'ajoute pas de puissance au signal modulé (cf. Eq. (9)).

\subsubsection{Cas d'un bruit blanc de fréquence dans une bande passante finie $B$}

Dans certaines situations expérimentales importantes, la fréquence laser et non la phase est modulée suivant un processus stochastique dans une bande passante $B$. Pour simplifier, nous supposerons que :

$$
S_{f}(\Omega)=\left\{\begin{array}{cc}
\frac{f_{m s}^{2}}{2 B}, & |\Omega| \leq 2 \pi B \\
0, & |\Omega|>2 \pi B
\end{array}\right.
$$

$\frac{f_{r m s}^{2}}{B}$ est la densité spectrale de bruit exprimée en $\mathrm{Hz}^{2} / \mathrm{Hz}$ et $\left(f_{r m s}^{2}\right)^{1 / 2}$ décrit la fluctuation moyenne en fréquence résultant de ce spectre de bruit. Selon Eq. (9), la densité spectrale de puissance de bruit de phase vaut $S_{\varphi}(\Omega)=\frac{S_{f}(\Omega)}{(\Omega / 2 \pi)^{2}}$ et diverge à basse fréquence. La densité spectrale de puissance du champ laser, si l'on néglige le bruit d'amplitude, est obtenue grâce aux équations (7) et (8) et par application du théorème de Wiener-Khintchine :

$$
S_{E}(\omega)=\int_{-\infty}^{+\infty} d \tau E_{0}^{2} \exp \left(i\left(\omega_{0}-\omega\right) \tau\right) \exp \left(-\left(C_{\varphi}(0)-C_{\varphi}(\tau)\right)\right)
$$

La fonction de $\tau$ dans l'intégrale (20) contribue à la densité de puissance essentiellement aux temps plus courts que l'inverse des excursions du bruit de fréquence, soit $\tau \leq\left(f_{r s s}^{2}\right)^{-1 / 2}$.

D'après Eq. (12) et utilisant à nouveau le théorème de Wiener-Khintchine, nous obtenons :

$$
\begin{aligned}
C_{\varphi}(0)-C_{\varphi}(\tau) & =\int_{-\infty}^{+\infty} \frac{d \Omega}{2 \pi} \frac{S_{f}(\Omega)}{(\Omega / 2 \pi)^{2}}(1-\exp (i \Omega \tau)) \\
& =\frac{2 \pi \tau f_{\operatorname{ms}}^{2}}{B} \int_{0}^{\pi B r} d \xi \frac{\sin ^{2}(\xi)}{\xi^{2}}
\end{aligned}
$$


Il faut noter que la fonction d'autocorrélation du bruit de phase, elle-même, diverge contrairement au cas du bruit blanc de phase. Seule, la différence $C_{\varphi}(0)-C_{\varphi}(\tau)$ conserve une valeur finie dont l'expression se simplifie pour les temps courts et les temps longs devant $1 / B$ :

$$
\begin{array}{r}
\text { Pour : } \pi B|\tau|<<1, C_{\varphi}(0)-C_{\varphi}(\tau) \approx 2 \pi^{2} f_{m s}^{2} \tau^{2} \\
\pi B|\tau| \gg>1, C_{\varphi}(0)-C_{\varphi}(\tau) \approx \frac{\pi^{2} f_{m s}^{2}}{B}|\tau|
\end{array}
$$

3.2.2.1 bruit basse fréquence : dans le cas où le bruit de fréquence est d'amplitude grande devant la bande passante, $\left(B<<\left(f_{r m s}^{2}\right)^{1 / 2}\right)$, alors l'expression (22) est applicable dans tout le domaine "utile" d'intégration, $\tau \leq\left(f_{\text {rms }}^{2}\right)^{-1 / 2}$, de l'Eq. (20). Finalement, la densité spectrale de puissance est une Gaussienne :

$$
S_{E}(\omega)=\frac{E_{0}^{2}}{\left(2 \pi f_{r m s}^{2}\right)^{1 / 2}} \exp \left(-\frac{\left(\omega-\omega_{0}\right)^{2}}{8 \pi^{2} f_{r m s}^{2}}\right)
$$

La largeur de raie laser à mi-hauteur est donnée par :

$$
\Delta v=\sqrt{\left[8 \ln (2) f_{r m s}^{2}\right]}
$$

Il s'agit typiquement de la forme de raie d'un laser à colorant.

3.2.2.2 bruit blanc de fréquence large bande : dans le cas où la bande passante est grande devant l'amplitude du bruit de fréquence $B \gg f_{m s}^{2}{ }^{1 / 2}$. l'essentiel du domaine "utile" d'intégration, $\tau \leq f_{m s}^{2-1 / 2}$, de l'Eq. (20) est situé dans la région $\pi \tau B \gg>1$ pour laquelle l'expression (23) s'applique. Finalement, la densité spectrale de puissance du champ laser est la transformée de Fourier d'une exponentielle, i.e. :

$$
S_{E}(\omega)=2 E_{0}^{2} \frac{\frac{\pi^{2} f_{r m s}^{2}}{B}}{\left(\omega-\omega_{0}\right)^{2}+\left(\frac{\pi^{2} f_{r m s}^{2}}{B}\right)^{2}}
$$

Il s'agit d'une Lorentzienne centrée à $\omega=\omega_{0}$ et de largeur à mi-hauteur :

$$
\Delta v=\frac{\pi f_{m s s}^{2}}{B}
$$

Cette forme de raie Lorentzienne est rencontrée dans deux situations importantes :

- le cas de la fréquence laser perturbée par l'émission spontanée coexistant inévitablement avec le processus d'émission stimulée responsable de l'effet laser (ainsi que les fluctuations du vide entrant dans la cavité). Elle donne lieu à un bruit blanc de fréquence et un bruit blanc d'amplitude. Ce dernier contribue en général de façon négligeable à la forme de raie qui résulte surtout du bruit blanc de fréquence. Il s'agit d'une forme de raie Lorentzienne (26) dont la largeur est appelée largeur de Schawlow-Townes (cf. cours "Bruit quantique dans les lasers" C. Fabre):

$$
\Delta v=\frac{D_{S T}}{2 \pi}=\frac{\kappa / 2 \pi}{\bar{N}}
$$


$\kappa$ est la largeur spectrale d'un mode de la cavité laser passive et $\bar{N}$ représente le nombre moyen de photons dans la cavité.

- le cas d'un laser stabilisé en fréquence dont le signal de correction est limité par le bruit de photons : nous reviendrons sur cette situation dans la quatrième section.

3.2.2.3 Remarque sur les largeurs de raies dans les deux cas ci-dessus : il n'est pas anodin que les dépendances des largeurs de raies avec le bruit de fréquence varie comme $\sqrt{f_{m x}^{2}}$ pour un bruit basse fréquence (Gaussienne) et comme $f_{m, t}^{2}$ pour un bruit large bande (Lorentzienne). En effet, lorsqu'on étudie le battement entre deux lasers identiques mais indépendants - ce qui est une procédure couramment employée pour caractériser les propriétés spectrales d'un laser -, dans le premier cas, la largeur de raie du battement (que l'on peut effectivement observer) est $\sqrt{2}$ fois la largeur de raie de chaque laser, dans le second cas, elle vaut 2 fois la largeur des lasers.

En effet, si $\varphi(t)=\varphi_{1}(t)-\varphi_{2}(t)$ est le bruit de phase du battement entre les lasers 1 et 2, la fonction d'autocorrélation, déduite de la définition (3), est simplement $C_{\varphi}(\tau)=C_{\varphi_{1}}(\tau)+C_{\varphi_{2}}(\tau)$ car les lasers sont supposés indépendants. D'après (4) et (12), les densités spectrales de puissances de bruit de fréquence s'ajoutent aussi : $S_{f}(\Omega)=S_{f_{1}}(\Omega)+S_{f_{2}}(\Omega)$, cqfd.

\subsubsection{Rôle du bruit basse fréquence :}

Un bruit blanc de phase sur le spectre de la fréquence laser se traduit par la réduction de la porteuse du champ laser et l'apparition d'un bruit blanc (cf. Eq. (16) et (17)). Un bruit blanc de fréquence a pour effet de faire disparaître toute porteuse de la forme de raie laser ; celle-ci présente, dès lors, une largeur finie (cf. Eqs. (25-27)), quelle que soit la bande passante de ce bruit. Ceci est une conséquence de la divergence de la densité de puissance de bruit de phase correspondante aux basses fréquences (comme $1 / \Omega^{2}$ ). En ce sens, le bruit de phase donne une indication sur le spectre du champ laser plus directe que le bruit de fréquence.

Néanmoins, expérimentalement, on a accès au bruit de fréquence d'un laser plus souvent qu'à son bruit de phase. Les signaux d'absorption en spectroscopie atomique ou moléculaire, les pics de transmission de cavités Fabry-Perot constituent des discriminateurs de fréquence qui permettent d'observer ce bruit de fréquence. C'est donc les composantes basse fréquence du bruit de fréquence qui jouent le rôle déterminant sur la largeur de raie laser. Ce fait est clairement illustré par les expressions (25) et (27) des largeurs de raie laser. Dans le cas d'un bruit basse fréquence, la largeur varie comme $f_{m s}^{21 / 2}$ (cf Eq. (25)), fluctuation moyenne en fréquence. Lorsque le bruit est large bande, la largeur varie comme $\frac{f_{m s}^{2}}{B} f_{m s}^{21 / 2}$ (cf Eq. (27)) où le rapport d'atténuation représente la fraction de bruit basse fréquence dans la bande passante $B$. A contrario, à un bruit blanc de phase correspond un bruit de fréquence qui diverge aux hautes fréquences comme $\Omega^{2}$. Ce bruit haute fréquence n'altère que de façon marginale le spectre du champ laser.

Ces considérations ont des conséquences importantes en matière de stabilisation en fréquence des lasers. En effet, un système d'asservissement en fréquence dont la bande passante est finie, pourra assurer une correction efficace des fluctuations aux basses fréquences. Par contre, il est fréquent que cela s'accompagne d'une légère dégradation du spectre au voisinage de la limite supérieure de la bande passante de l'asservissement. Ceci n'empêchera pas un affinement significatif de la raie laser si du moins la bande passante de l'asservissement est comparable ou supérieure à l'amplitude des fluctuations du laser "libre". 


\subsubsection{Bruit très basse fréquence et temps fini de la mesure}

La description ci-dessus supposait implicitement que l'on avait affaire à des phénomènes stationnaires i.e. la fréquence laser oscille autour d'une fréquence $\omega_{0}$, fixe au cours du temps. Expérimentalement, ceci n'est jamais vérifié car les expériences s'effectuent sur un temps fini et une fréquence laser même asservie est susceptible de dériver de façon irréversible. Une dérive peut être interprétée comme un bruit de fréquence nulle d'amplitude infinie. De ce fait, la largeur de raie laser est strictement infinie. Il est vrai que le signal d'absorption accumulé sur un temps très long se trouvera fortement élargi sous l'effet d'une telle dérive. Heureusement, il est tout de même possible d'obtenir une résolution finie. Pour obtenir une largeur de raie laser significative pour la résolution de l'expérience, on peut introduire une fonction d'autocorrélation modifiée:

$$
\bar{C}_{E}(\tau)=\frac{1}{2 T} \int_{-T}^{T} d t E(t+\tau) E^{*}(t)
$$

où $[-T, T]$ est l'intervalle de temps dans lequel se déroule l'expérience. Dans ce cas, la nouvelle fonction d'autocorrélation est non nulle seulement pour $|\tau|<2 T$. La forme de raie laser effective est obtenue en modifiant l'équation (13) :

$$
\bar{S}_{E}(\omega)=\int_{-\infty}^{+\infty} d \tau \bar{C}_{E}(\tau) \exp (-i \omega \tau)
$$

Considérons comme seule source de bruit une dérive linéaire en fréquence $\omega=\omega_{0}+\alpha \tau$ soit $E(t)=E_{0} \exp \left(i \int_{0}^{t} d t^{\prime} \omega\left(t^{\prime}\right)\right)$, pour $-T<t<T$. On démontre aisément le résultat intuitif suivant :

- si la dérive est faible pendant le temps de mesure $(\alpha T \ll 1 / T)$, la largeur spectrale est imposée par $1 / T$ :

$$
\bar{S}_{E}(\omega)=4 E_{0}^{2} \frac{\sin ^{2}\left(\left(\omega-\omega_{0}\right) T\right)}{\left(\omega-\omega_{0}\right)^{2} T}
$$

ce spectre tend bien vers un pic de Dirac si le temps de mesure est infini.

- si la dérive est importante pendant le temps de mesure $(\alpha T \gg 1 / T)$, la largeur spectrale est imposée par cette dérive. Le spectre laser est "vu" comme un spectre blanc de largeur égal à la dérive pendant le temps de mesure :

$$
\bar{S}_{E}(\omega)=\frac{E_{0}^{2}}{2 \alpha T},-\alpha T<\omega-\omega_{0}<\alpha T
$$

En fait, le temps fini de la mesure ou de l'expérience opère comme un filtre passe-haut $[1 / T,+\infty[$ pour le bruit de fréquence du spectre lumineux qui, par contre, sera "vu" avec une largeur égale à l'inverse de ce temps de mesure augmentée par le bruit "haute fréquence" présent sur le spectre. C'est pourquoi, dans les expériences où la résolution spectrale est visée, il existera un temps de mesure optimal.

\subsection{Variance d'Allan}

La description du champ laser donnée ci-dessus est effectuée dans le domaine fréquentiel. Une description équivalente dans le domaine temporel est bien entendu possible. 


\subsubsection{Définition :}

La fréquence laser instantanée est définie par :

$$
v(t)=v_{0}+\frac{1}{2 \pi} \frac{d \varphi}{d t}=v_{0}(1+y(t))
$$

La fonction $y(t)$ est une grandeur sans dimension qui représente les fluctuations relatives de fréquence laser. Expérimentalement, on peut mesurer la fréquence laser (ou du moins le battement entre deux lasers) à l'aide d'un compteur sur un laps de temps fini, $\tau$. Une telle mesure peut être effectuée à intervalles réguliers $T$. Soit $t_{k}$ le début de la kième mesure, alors :

$$
\langle v(t)\rangle_{t_{t}, \tau}=v_{0}+\frac{1}{\tau} \int_{t_{t}}^{t_{t}+\tau} d t \frac{1}{2 \pi} \frac{d \varphi}{d t}=v_{0}\left(1+\bar{y}_{k, \tau}\right)
$$

On définit alors la variance de $y(t)$ à $N$ échantillons :

$$
\sigma_{y}^{2}(N, T, \tau)=\frac{1}{N-1} \sum_{i=1}^{N}\left(\bar{y}_{i, \tau}-\frac{1}{N} \sum_{j=1}^{N} \bar{y}_{j, \tau}\right)^{2}
$$

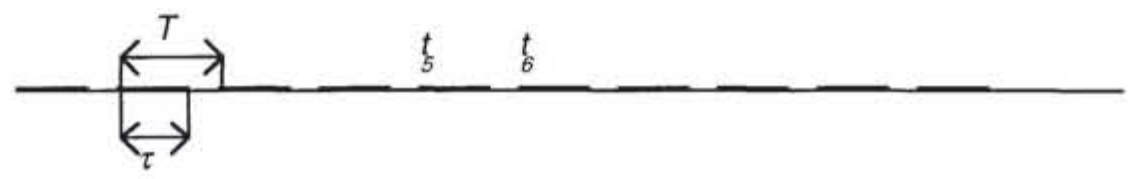

Figure 5 : représentation schématique des deux temps caractéristiques de la variance $\sigma_{y}^{2}(N, T, \tau)$

La variance à deux échantillons et telle que $T=\tau$ joue un rôle particulier. Si on moyenne cette variance sur un très grand nombre d'échantillons, on obtient la variance d'Allan, soit :

$$
\sigma_{y}^{2}(\tau)=\left\langle\sigma_{y}^{2}(2, T, \tau)\right\rangle=\frac{1}{2}\left\langle\left(\bar{y}_{2, \tau}-\bar{y}_{1, \tau}\right)^{2}\right\rangle
$$

En pratique, le nombre de mesures $m$ est fini. La variance d'Allan est approchée par l'expression :

$$
\sigma_{y}^{2}(\tau) \approx \frac{1}{m} \sum_{k=1}^{m} \frac{\left(\bar{y}_{k+1, \tau}-\bar{y}_{k, \tau}\right)^{2}}{2}
$$

avec une précision :

$$
\frac{\Delta \sigma^{2}}{\sigma^{2}} \approx \frac{1}{\sqrt{m}}
$$

Expérimentalement, on accède à la variance d'Allan d'un laser en mesurant le battement avec un laser de référence beaucoup plus stable. Si l'on dispose de deux lasers identiques et indépendants, une bonne estimation de la variance d'Allan de chaque laser est donnée par la variance du battement des lasers divisé par deux.

\subsubsection{Relation entre variance d'Allan et densité spectrale :}

D'après Eq. (33), les densités spectrales de puissance associées au bruit de fréquence et à $y(t)$ sont reliées par: 


$$
S_{f}(\Omega)=v_{0}^{2} S_{y}(\Omega)
$$

Les informations apportées par la variance d'Allan et par la densité spectrale de puissance sont équivalentes, du moins lorsque celle-ci est effectivement définie. Ceci est exprimé par le théorème de Cutler (cf Annexe) :

$$
\sigma_{y}^{2}(\tau)=\frac{1}{\pi} \int_{-\infty}^{+\infty} d \Omega S_{y}(\Omega) \frac{\sin ^{4}\left(\frac{\Omega \tau}{2}\right)}{\left(\frac{\Omega \tau}{2}\right)^{2}}
$$

Expérimentalement, on rencontre différents types de bruit qui varient selon une puissance de la fréquence :

$$
S_{y}^{\prime}(\Omega)=h_{l}(|\Omega| / 2 \pi)^{\prime}
$$

La variance d'Allan est alors reliée à la densité spectrale de bruit de fréquence dans les cas importants suivants :

\begin{tabular}{|c|c|c|c|}
\hline$l$ & $S_{y}^{l}(\Omega)$ & $\sigma_{y}^{2}(\tau)$ & type de bruit \\
\hline-2 & $h_{-2}(\Omega / 2 \pi)^{-2}$ & $h_{-2} \frac{4 \pi^{2}}{3} \tau$ & $\begin{array}{c}\text { Marche aléatoire de } \\
\text { fréquence }\end{array}$ \\
\hline-1 & $h_{-1}(|\Omega| / 2 \pi)^{-1}$ & $h_{-1} 4 \ln (2)$ & Bruit de Flicker \\
\hline 0 & $h_{0}$ & $h_{0} \frac{1}{\tau}$ & $\begin{array}{c}\text { Bruit blanc de } \\
\text { fréquence }\end{array}$ \\
\hline
\end{tabular}

Tableau I : Relation entre densité spectrale et variance d'Allan pour différents types de bruit.

Dans le cas d'une dérive linéaire de la fréquence, la densité spectrale n'est pas définie. Par contre, une simple application de l'équation (36) montre que la variance d'Allan est bien définie et est proportionnelle à $\tau^{2}$.

Le mode de représentation o le plus utilisé est une représentation de la racine carré de la variance d'Allan en fonction du temps en échelle Log-Log.

La Figure 6 représente une allure typique et schématique d'une variance d'Allan en présence de différents types de bruits:

- le bruit blanc en fréquence, dominant à haute fréquence. Il se traduit par une pente -1/2. Les relations du Tableau 1 et les expressions (19) et (27) montrent que la largeur de raie laser est reliée à la variance d'Allan par:

$$
\Delta v=2 \pi v_{0}^{2} \sigma_{y}^{2}(\tau) \tau, \tau<\tau_{0}
$$

- A partir d'un temps $\tau=\tau_{0}$, il apparaît un plateau dit "plancher de Flicker" qui correspond à un bruit basse fréquence en $1 / \mathrm{f}(=2 \pi / \Omega)$. Ce type de bruit est rencontré sur pratiquement tous les oscillateurs, son origine physique n'est pas très bien comprise. Ce plancher joue un rôle important puisqu'il correspond à la précision maximale avec laquelle la fréquence de l'oscillateur peut être connue, quel que soit le temps d'intégration de la mesure. 


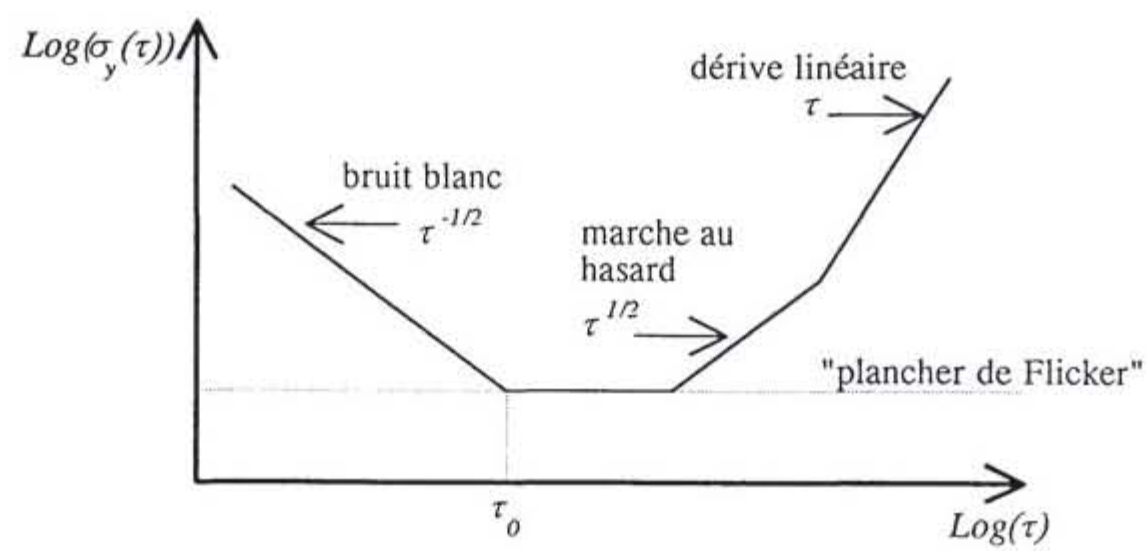

Figure 6: Représentation de la variance d'Allan d'un oscillateur en échelle Log-Log, en présence de quelques types de bruit.

- Sur des temps encore plus longs, des bruits de plus basse fréquence peuvent devenir dominants. Il peut s'agir de bruits tels que la fréquence évolue suivant un mouvement brownien (marche au hasard de pente $1 / 2$ ), ou linéairement avec le temps (pente 1), etc. Il s'agit de systèmes dont la fréquence diverge : c'est le cas d'un laser libre, ce peut être le cas d'un laser asservi sur une référence de fréquence qui, elle-même, dérive. La plage de temps sur laquelle une mesure de fréquence sera la plus précise correspond avec celle où le "plancher de Flicker" est atteint.

\section{STABILISATION EN FRÉQUENCE}

Les méthodes de stabilisation en fréquence des lasers couvrent un domaine extrêmement varié. Il est hors de propos d'en faire un catalogue. Nous nous attacherons à présenter le principe d'un asservissement ainsi que les conditions à réunir pour que celui-ci soit efficace.

\subsection{Principe de l'asservissement}

La stabilisation en fréquence ou en phase d'un oscillateur (laser) met en jeu plusieurs éléments :

- Le champ laser porteur d'un bruit de fréquence ou de phase dont la composante de Fourier à $\Omega$ avant asservissement est $\varepsilon(\Omega)$.

- Une référence de fréquence ou de phase.

- Un comparateur qui a pour but de mesurer l'erreur par rapport à la référence.

- Un transducteur qui permet d'appliquer le signal de correction élaboré à partir du signal d'erreur après amplification et filtrage (la fonction de transfert correspondante est $G(\Omega)$ ).

Figure 7 représente le principe de fonctionnement de l'asservissement :

Le signal d'erreur résiduel en boucle fermée, $\varepsilon_{s}(\Omega)$, satisfait à la relation suivante :

$$
\varepsilon_{s}(\Omega)=\varepsilon(\Omega)-G(\Omega) \varepsilon_{s}(\Omega) \Rightarrow \varepsilon_{s}(\Omega)=\frac{\varepsilon(\Omega)}{1+G(\Omega)}
$$




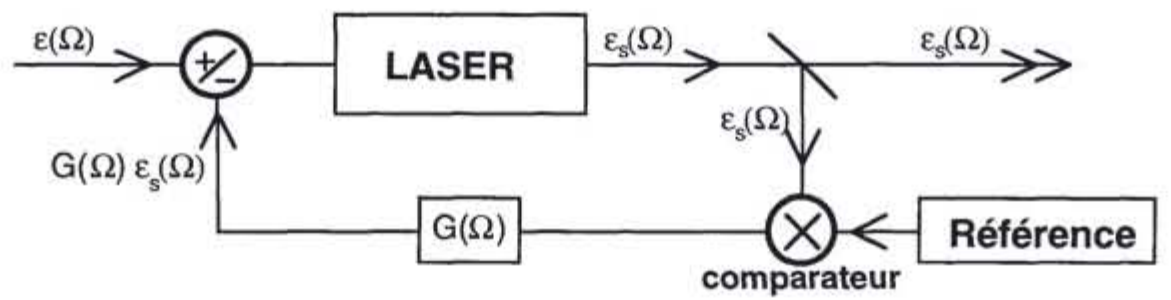

Figure 7 : Schéma de principe d'un asservissement. $\varepsilon(\Omega)$ est l'erreur de phase/fréquence en boucle ouverte à la fréquence $\Omega$. $\varepsilon_{s}(\Omega)$ est l'erreur résiduelle après correction.

Sur le schéma de la figure 7, le laser est placé à l'intérieur de la boucle d'asservissement. Il est également possible d'utiliser un laser libre (ou stabilisé sur le long terme seulement) et d'effectuer les corrections directement sur le faisceau de sortie à l'aide de transducteurs comme des modulateurs acousto-optiques et électro-optiques. Les deux méthodes peuvent enfin être combinées.

Comme le gain de l'asservissement à fréquence nulle, $G(0)$, est nécessairement fini, il en résulte une erreur statique résiduelle. Un asservissement en phase se caractérise essentiellement par le fait que l'on mesure la phase du laser par rapport à une référence de phase. Par exemple, l'injection d'un laser dans un second laser réalise la mise en phase du second laser sur le premier. Le battement entre deux lasers peut lui-même être mis en phase sur une source radiofréquence très stable. Les deux lasers acquièrent alors une relation de phase bien définie. Il y aura dans ce cas une erreur statique de phase. Par contre, il n'y a pas d'erreur sur la fréquence moyenne contrairement à l'asservissement en fréquence où l'on effectue une comparaison de fréquence.

Dans la suite de ce cours, nous allons principalement discuter des asservissements en fréquence et de l'influence des différents éléments constitutifs.

\subsection{Les références de fréquence et leurs limitations}

\subsubsection{Les références de fréquence}

Les propriétés spectrales du laser à stabiliser ne pourront, en général, pas être meilleures que celles de la référence de fréquence utilisée dans l'asservissement.

4.2.1.1 Résonance d'un interféromètre Fabry-Perot : un premier type de référence est constitué par un résonateur optique. Par exemple, une cavité Fabry-Perot symétrique constituée de deux miroirs de rayon de courbure $R$ et séparés d'une distance $L_{O}$ présente une série de fréquences de résonance, $v_{\text {lmn }}$, données par:

$$
v_{l m n}=\frac{c}{2 L}\left[n+1+\frac{1}{\pi}(l+m+1) \cos ^{-1}\left(1-\frac{L}{R}\right)\right]
$$

où $L$ désigne la longueur optique associée à $L_{0}$.

Une telle référence pose deux problèmes. Elle ne peut servir de calibration de la fréquence absolue car celle-ci dépend de la longueur de la cavité qui n'est pas suffisamment bien connue. Elle ne présente pas un caractère de stabilité intrinsèque car la longueur de la cavité dérive au cours du temps sous l'effet de la dilatation thermique, par exemple. Il est néanmoins possible de prendre de grandes précautions dans la réalisation de la cavité. On a ainsi pu réduire les causes de dérive de façon telle que le vieillissement ("aging") du matériau utilisé (U.L.E., ultra-low expansion material) reste le facteur dominant de dérive. Une dérive très linéaire (donc 
prédictible) de l'ordre d'un $\mathrm{Hz} / \mathrm{s}$ dans le visible a ainsi pu être obtenu. Ceci est une stabilité bien souvent suffisante.

4.2.1.2 Transition atomique ou moléculaire : on peut considérer une fréquence de transition atomique ou moléculaire car elle ne dépend que de constantes dites fondamentales, masses, charge électrique,... supposées invariables. Il existe cependant des modèles théoriques prédisant des variations de certaines constantes au cours du temps. Un test expérimental consisterait à observer une dérive dans la mesure de l'écart entre deux fréquences atomiques dépendant de ces constantes de façon différente au cours du temps.

En l'absence d'une telle observation qui semble, pour l'instant, hors de portée de l'expérience, ces fréquences de transition constituent des références de fréquence idéales, car elles possèdent une stabilité intrinsèque. Le standard primaire de temps/fréquence est actuellement une transition radio-fréquence de l'atome de césium à $9 \mathrm{GHz}$. La mesure de fréquence d'une transition atomique ou moléculaire située dans le domaine optique nécessite la réalisation de chaînes de fréquence pour raccorder la région spectrale considérée au standard primaire de fréquence. Dès le début des années 70 , des mesures de fréquences absolues dans le domaine spectral de $10 \mu \mathrm{m}$ (lasers à $\mathrm{CO}_{2}$ ) ont été effectuées, donnant lieu aux premiers standards (secondaires) de fréquence optique. Depuis peu, des mesures de fréquences dans le domaine visible ont été réalisées. Auparavant, on accédait à la fréquence via une mesure interférométrique de la longueur d'onde, méthode potentiellement bien moins précise qu'une mesure de fréquence.

\subsubsection{Réalisation de la référence de fréquence et élaboration du signal d'erreur}

4.2.2.1 Résonance atomique ou moléculaire : le standard de fréquence basé sur une transition atomique ou moléculaire correspond à l'écart de fréquence entre deux niveaux d'énergie du système supposé isolé et au repos. C'est la seule définition absolue possible.

Toute réalisation pratique d'un tel standard est incompatible avec cette définition et risque d'introduire des erreurs "systématiques" de la fréquence. Trois effets sont inévitablement présents :

- les déplacements lumineux dus à la présence du champ laser indispensable à la détection du signal.

- le déplacement collisionnel dû à l'environnement.

- l'effet Doppler du second ordre, $\Delta \omega=-\omega_{0} \frac{v^{2}}{2 c^{2}}$, qui déplace la résonance de l'atome en mouvement sondée par le laser (l'effet Doppler du premier ordre est, en moyenne, nul).

Ces erreurs systématiques doivent être minimisées, elles sont parfois mesurées notamment grâce à une étude fine de la forme de raie et, dans ce cas, peuvent être partiellement compensées. Elles constituent une limite à l'exactitude de la fréquence.

Le type d'expérience effectué pour élaborer le signal d'erreur de la fréquence laser à asservir peut induire d'autres erreurs de nature plus instrumentale, telle que la courbure des fronts d'ondes ou la distorsion par modulation. On peut néanmoins énoncer une règle empirique : les effets systématiques sont une fraction, petite en général, de la largeur de la résonance détectée, c'est pourquoi un progrès significatif de l'exactitude d'un standard de fréquence accompagne en général un gain dans le pouvoir de résolution de l'expérience qui réalise ce standard. C'est le cas de l'horloge à césium réalisée à l'aide d'une fontaine d'atomes de césium refroidis.

Ces erreurs affectent la fréquence moyenne à laquelle est asservi le laser. La largeur de raie laser ultime en présence d'un asservissement idéal dépend, quant à elle, du rapport signal-surbruit avec lequel est obtenu le signal d'erreur de fréquence. Considérons une expérience d'absorption saturée (i.e. signal de résonance non élargi par effet Doppler provenant des seuls atomes ou molécules se propageant perpendiculairement à une onde stationnaire du laser).

La détection du signal démodulé à une harmonique impaire de la fréquence de modulation, $\mathrm{f}_{\mathrm{m}}$, donne lieu à un signal de forme impaire par rapport à la fréquence de résonance. Il peut donc jouer le rôle de discriminateur de fréquence. Le choix de la fréquence et la profondeur de modulation est gouverné par la largeur de raie. La détection à une harmonique élevée à pour 
effet de réduire le contraste des signaux parasites larges présents avec le signal de saturation (absorption linéaire, profil de gain du laser, franges d'interférence ,...). Ceux-ci introduiront des offsets sur le point de fonctionnement de l'asservissement, qui peuvent dériver au cours du temps.

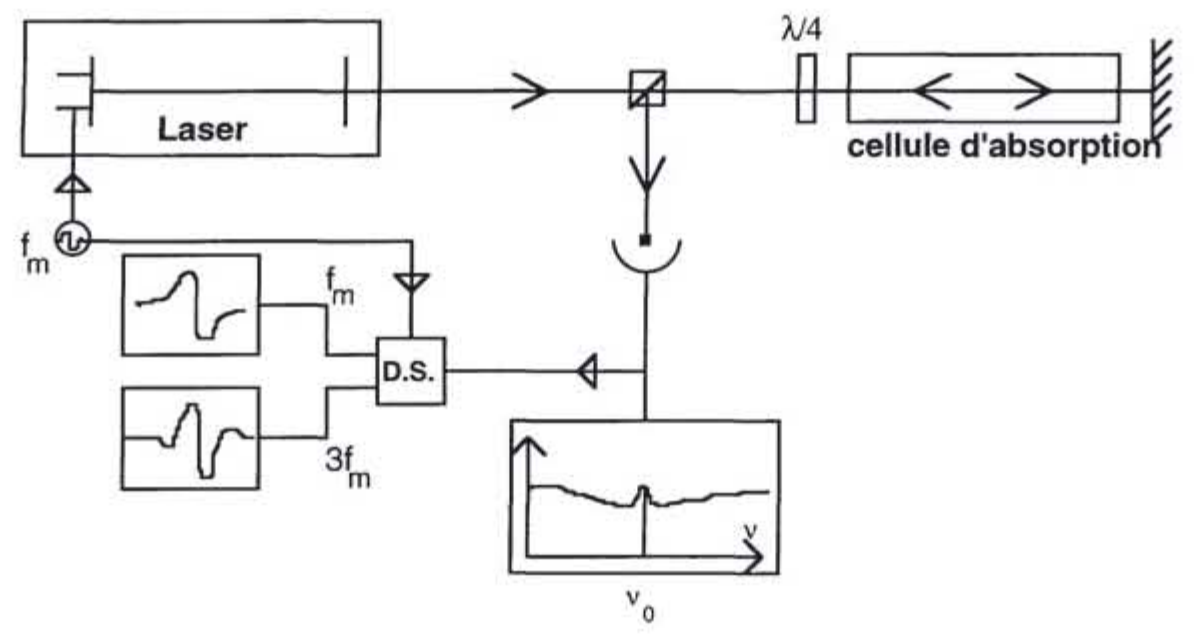

Figure 8 : Schéma d'une expérience d'absorption saturée utilisée pour obtenir un discriminateur de fréquence propice à l'asservissement en fréquence du laser. les signaux démodulés à $\mathrm{f}_{\mathrm{m}}$ et $3 \mathrm{f}_{\mathrm{m}}$ présentent la symétrie adéquate.

Le bruit d'amplitude présent sur le signal comporte souvent une partie basse fréquence en $1 / f$, dit bruit technique. On choisit si possible la fréquence de détection du signal d'erreur hors de la bande passante de ce bruit, ce qui peut être réalisé par la détection de la troisième harmonique de la fréquence de modulation. Dans la partie haute fréquence, il reste un bruit blanc. Celui-ci aura couramment deux origines : le bruit du détecteur indépendant de la puissance laser et le bruit de photons (ou shot noise) qui varie comme la racine carré de la puissance laser. Ce bruit d'amplitude présent sur le signal discriminateur de fréquence ne peut être distingué des fluctuations de fréquence et, de ce fait, sera interprété comme un bruit de fréquence par la boucle d'asservissement. Cette erreur d'interprétation inévitable est responsable de la largeur de raie finie en deçà de laquelle il ne sera pas possible de descendre même avec un asservissement de gain infini.

Nous allons calculer cette largeur dans le cas où le bruit dominant est le bruit de photons. Soient $P_{L}$, la puissance laser incidente sur la photodiode de rendement quantique $\eta, i$, le photocourant ainsi généré, $i_{s}$, le courant associé au signal de saturation et $i_{s . n}$, le courant de bruit de photons produit dans la bande passante de la détection, $B$. Nous avons les relations suivantes:

$$
\begin{aligned}
& i=e \eta \frac{P_{L}}{\hbar \omega}=\rho P_{L} \\
& i_{s}=\rho C P_{L} \\
& i_{s n}=\sqrt{2 e i B}
\end{aligned}
$$


Dans Eq. (46), C désigne le contraste du signal. Le courant de bruit de photons varie comme la racine carré de la bande passante car c'est la puissance de bruit qui lui est proportionnelle dans le cas d'un bruit blanc. Le signal d'erreur, en présence du bruit d'amplitude, est représenté sur la Figure 9:

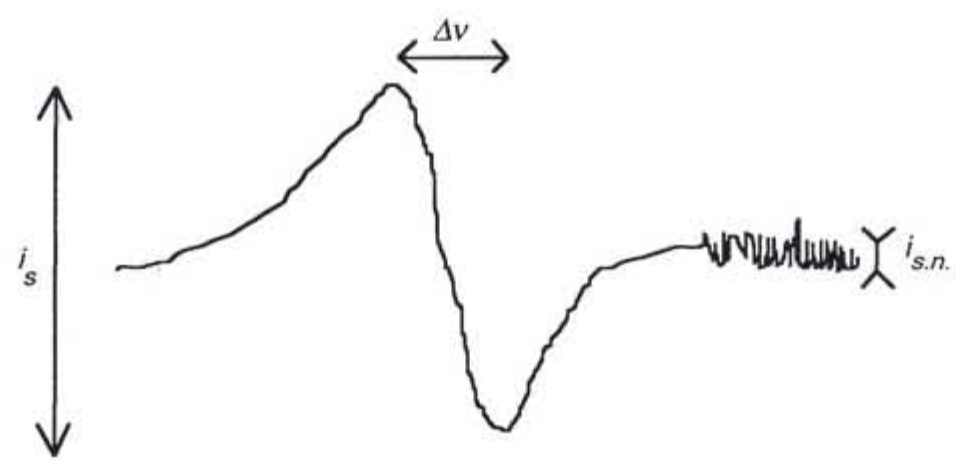

Figure 9 : Le bruit d'amplitude, $i_{s . n}$, du laser présent sur le signal d'erreur est mesurable sur la ligne de base de ce signal. Egalement présent au centre du discriminateur de fréquence, il s'ajoute sans distinction possible au bruit lié aux fluctuations de fréquence que l'on cherche à corriger.

Le bruit de fréquence ramené par un asservissement parfait à cause du bruit d'amplitude est donné par:

$$
f_{m s}^{2}=\left(i_{s . n .} \frac{\Delta v}{i_{s}}\right)^{2}
$$

Ce bruit de fréquence reproduit les propriétés spectrales du bruit d'amplitude. Dans le cas d'un bruit blanc, la raie laser tendra vers une Lorentzienne dont la largeur se déduit de l'Eq. (27), soit :

$$
\delta v=2 \pi \frac{(\Delta v)^{2}}{C^{2} \eta P_{L} / \hbar \omega}
$$

A.N. : $\Delta v=100 \mathrm{kHz}, C=5 \cdot 10^{-3}, \eta=0.5, P_{L}=1 \mathrm{~mW}, v=30 \mathrm{THz} \Rightarrow \delta v=0.1 \mathrm{~Hz}$.

Les paramètres de puissance, contraste et largeur de raie sont interdépendants à cause des effets de saturation et dépendent eux-mêmes des paramètres atomiques et moléculaires. Il existe donc un optimum des paramètres expérimentaux et la largeur de raie laser ultime dépend de façon sensible de la transition atomique ou moléculaire choisie.

4.2.2.2 Résonance d'une cavité Fabry-Perot : la stabilisation sur une résonance d'une cavité Fabry-Perot ne présente pas ce type de limitation car l'interaction est essentiellement linéaire. C'est pourquoi on choisit parfois ce type de discriminateur de fréquence pour affiner la raie laser, la référence atomique ou moléculaire servant alors à la stabilité à long terme.

Le montage expérimental typique est le suivant : 


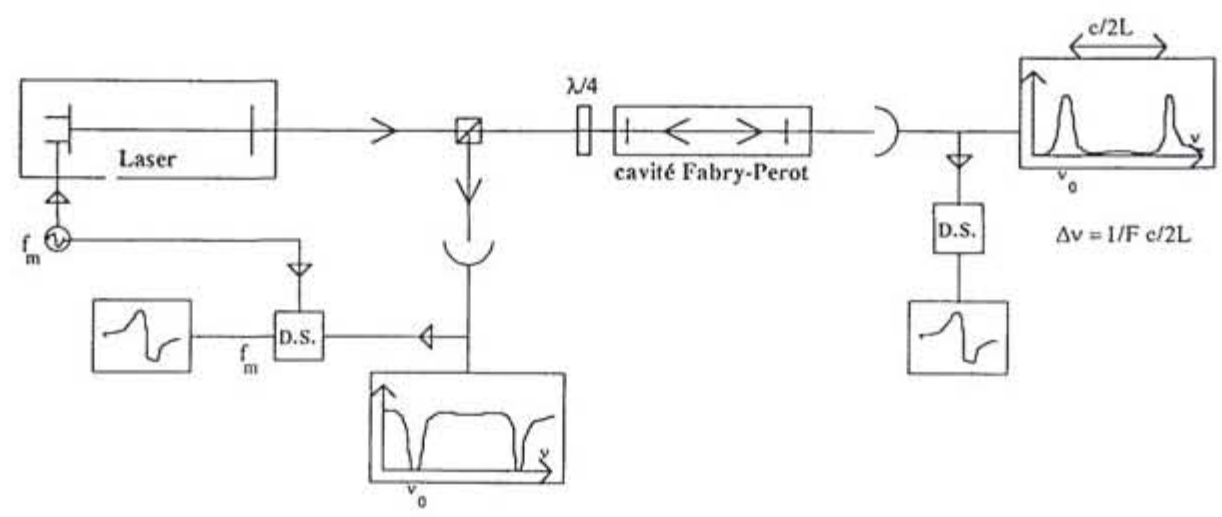

Figure 10 : Principe du montage permettant l'asservissement en fréquence sur le pic de transmission ou le pic de réflexion d'une cavité Fabry-Perot.

Il est possible d'asservir la fréquence laser sur le pic de transmission ou le pic de réflexion. Le calcul de la largeur de raie laser ultime pour un asservissement sur le pic de transmission est similaire au cas d'une résonance atomique. Si le bruit dominant est le bruit de photons, on trouve :

$$
\delta v=2 \pi \frac{(\Delta v)^{2}}{\eta P_{L} / \hbar \omega}
$$

A.N. : $\Delta v=500 \mathrm{kHz}, \eta=0.5, P_{L}=1 \mathrm{~mW}, v=30 \mathrm{THz} \Rightarrow \delta v=50 \mu \mathrm{Hz}$.

L'expression (50) est la même que (49) dans laquelle $C=1$. L'autre différence vient du fait qu'il n'y a pas de saturation et si la puissance laser incidente augmente, la largeur de raie ultime continue à diminuer car le signal continue à croître sans élargissement. C'est pourquoi les largeurs de raies ultimes peuvent être extrêmement étroites. En pratique, le gain et la bande passante de l'asservissement seront les facteurs limitants.

Si l'asservissement est réalisé sur le pic réfléchi, le bruit d'amplitude du laser est nul à la résonance car le signal continu tombe à zéro. Dans ce cas, le bruit du détecteur est le seul bruit présent. Il s'agit d'un bruit blanc indépendant de la puissance laser. Par suite, la largeur de raie ultime, $\delta v$, est proportionnelle à $1 / P_{L}^{2}$.

\subsection{La boucle d'asservissement et ses limitations}

Dans la section précédente, nous avons vu les limites apportées par le bruit présent dans la détection de la référence de fréquence. Ici, nous faisons abstraction de ces limitations et considérons celles imposées par la boucle d'asservissement elle-même. Pour cela, nous allons considérer à nouveau un asservissement en fréquence d'un laser présentant un bruit blanc de fréquence:

$$
S_{f}(\Omega)=\frac{b}{2}
$$




\subsection{Boucle constituée d'un intégrateur et d'un gain proportionnel}

Nous avons vu que le bruit basse fréquence joue le rôle dominant dans la largeur de raie. C'est pourquoi un système d'asservissement, pour être efficace, devra présenter un gain très élevé dans ce domaine-là. Un intégrateur est le cas le plus simple que l'on puisse considérer. On peut lui ajouter un gain proportionnel qui corrigera également le bruit plus haute fréquence. La fonction de transfert d'une boucle d'asservissement constituée d'un intégrateur et d'un gain proportionnel est :

$$
G(\Omega)=K_{p}+\frac{1}{i \Omega \tau_{i}}=K_{p}+\frac{\Omega_{i}}{i \Omega}
$$

L'analogue électrique d'une telle fonction de transfert est donnée par un amplificateur opérationnel comme ci-dessous :
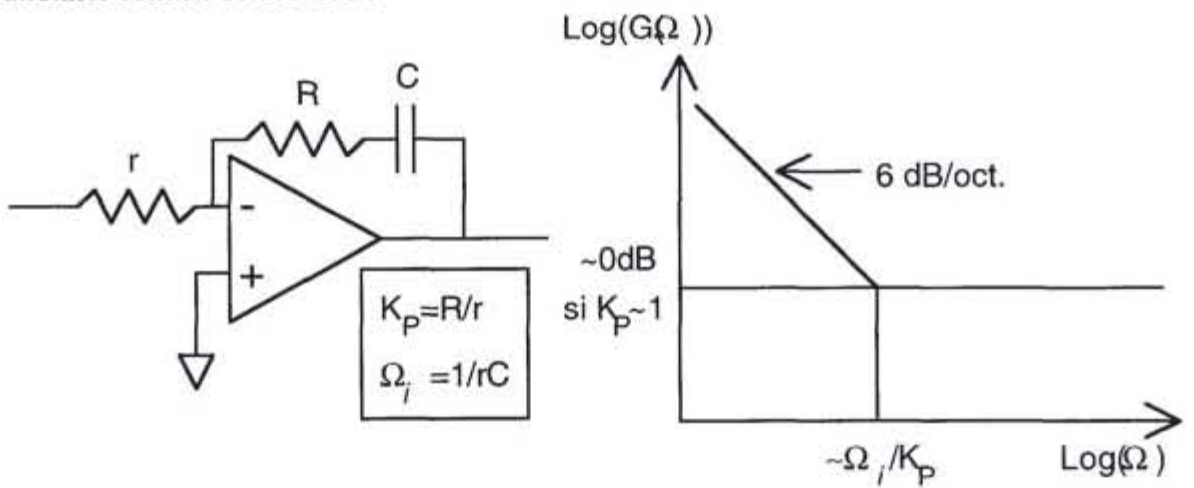

Figure 11 : Fonction de transfert en échelle Log-Log d'un intégrateur et d'un gain proportionnel (à droite) et l'analogue électrique d'un montage ayant une telle fonction de transfert (à gauche). (43):

En boucle fermée, la densité spectrale de puissance de bruit de fréquence devient selon Eq.

$$
S_{f}^{\text {stub. }}(\Omega)=\frac{S_{f}(\Omega)}{|1+G(\Omega)|^{2}}=\frac{b}{2} \frac{\Omega^{2}}{\Omega_{i}^{2}+\left(K_{p}+1\right)^{2} \Omega^{2}}
$$

On utilise successivement les équations (12) et la réciproque de (4), pour obtenir :

$$
C_{\varphi}(0)-C_{\varphi}(\tau)=b^{\prime}\left(1-\exp \left(-\frac{\Omega_{i}|\tau|}{K_{p}+1}\right)\right)
$$

où $b^{\prime}=\frac{b}{\left(K_{p}+1\right) \Omega_{i}}$. La forme de raie du laser asservi se déduit alors de Eq. (20) en effectuant le développement en série de la fonction exp :

$$
\begin{aligned}
S_{E}(\omega)= & 2 \pi E_{0}^{2} \exp \left(-b^{\prime}\right) \delta\left(\omega-\omega_{0}\right) \\
& +2 E_{0}^{2} \exp \left(-b^{\prime}\right) \sum_{i}^{+\infty} \frac{b^{\prime n}}{n !} \frac{\frac{n \Omega_{i}}{K_{p}+1}}{\left(\omega-\omega_{0}\right)^{2}+\left(\frac{n \Omega_{i}}{K_{p}+1}\right)^{2}}
\end{aligned}
$$


Cette expression montre que c'est l'intégrateur qui permet de rapporter de l'énergie dans la porteuse (si $\Omega_{q} \neq 0$ ). En l'absence d'intégrateur le niveau de bruit blanc en fréquence est simplement divisé par $\left(K_{P}+1\right)^{2}$. Le gain d'un intégrateur réel sature nécessairement. Il en résultera une porteuse légèrement élargie par cette saturation.

\subsubsection{Le délai dans les asservissements}

La description ci-dessus suppose que la correction est appliquée de façon instantanée. Ceci n'est jamais vrai : il faut prendre en compte le temps de réponse des différents éléments de chaîne de mesure. En dernier ressort, le temps de propagation fini de la lumière le long du trajet optique utilisé pour l'élaboration du signal de correction est le délai minimum qu'il faut considérer. Par exemple, il inclut le temps de stockage de la lumière dans une cavité Fabry-Perot si l'asservissement est réalisé sur le pic de transmission. Intuitivement, on comprend qu'il ne sera pas possible de corriger à des fréquences supérieures à $1 / \tau$ si $\tau$ désigne ce délai. On peut ainsi montrer dans le cas de l'intégrateur et du gain proportionnel que le critère de stabilité de l'asservissement sera stable si :

$$
\frac{\Omega_{i} \tau}{\sqrt{1-K_{P}^{2}}}<\frac{\pi}{2}+\operatorname{Arcsin}\left(K_{P}\right) \text { et } 0 \leq K_{P}<1
$$

En pratique, il est préférable d'introduire dans la fonction de transfert une fréquence de coupure (filtre passe-bas) inférieure à l'inverse de ce délai afin de pouvoir augmenter le gain proportionnel et de corriger de façon optimale le bruit de fréquence.

\section{EXEMPLES}

Pour illustrer les résultats établis dans les deux parties précédentes, nous allons donner deux exemples d'expériences réalisées pour démontrer soit les propriétés spectrales des lasers soit l'efficacité que l'on peut atteindre par des méthodes d'asservissement rapide.

\subsection{Asservissement d'un laser à colorant sur une cavité Fabry-Perot (d'après [8])}

\subsection{1 le laser libre}

Un laser à colorant présente un bruit de fréquence important. Celui-ci résulte notamment des fluctuations d'épaisseur du jet de colorant circulant au travers de la cavité. Si l'on considère une cavité de longueur $1 \mathrm{~m}$ et un changement d'épaisseur du jet de colorant de l'ordre de $1 \mathrm{~nm}$ (quelques couches moléculaires), dont l'indice est de l'ordre de 2.5 , le changement de fréquence de résonance de la cavité laser ainsi induit à $600 \mathrm{THz}(\tau=0.5 \mu \mathrm{m})$ vaut $900 \mathrm{kHz}$. La technologie des jets et des buses permet difficilement de descendre en dessous de ce niveau de fluctuations pour le laser libre. Ce bruit de fréquence est un bruit basse fréquence (faible devant les excursions de fréquences $(900 \mathrm{kHz})$ ) qui s'étend au plus sur quelques dizaines de $\mathrm{kHz}$. Pour connaître la forme de raie du laser libre, il suffira d'enregistrer avec un analyseur de spectre radio-fréquence le signal de battement entre ce laser et un laser de plus haute pureté spectrale comme un laser HeNe. Pour s'affranchir des effets de dérive de la fréquence du laser libre. Il suffit d'asservir la fréquence du laser avec une bande passante très faible de façon à stabiliser sur le long terme la fréquence moyenne sans modifier la pureté spectrale (fluctuations à court terme).

Ce laser libre correspond au cas d'un oscillateur en présence d'un bruit basse fréquence important dont la forme de raie peut être modélisée par une Gaussienne (cf 3.2.2.1). La Figure 
12 montre le profil de raie modélisé par une Gaussienne de largeur $450 \mathrm{kHz}$. Ceci correspond, d'après Eq. (25), à une excursion moyenne de bruit de fréquence de $\sqrt{f_{m s}^{2}} \approx 190 \mathrm{kHz}$.

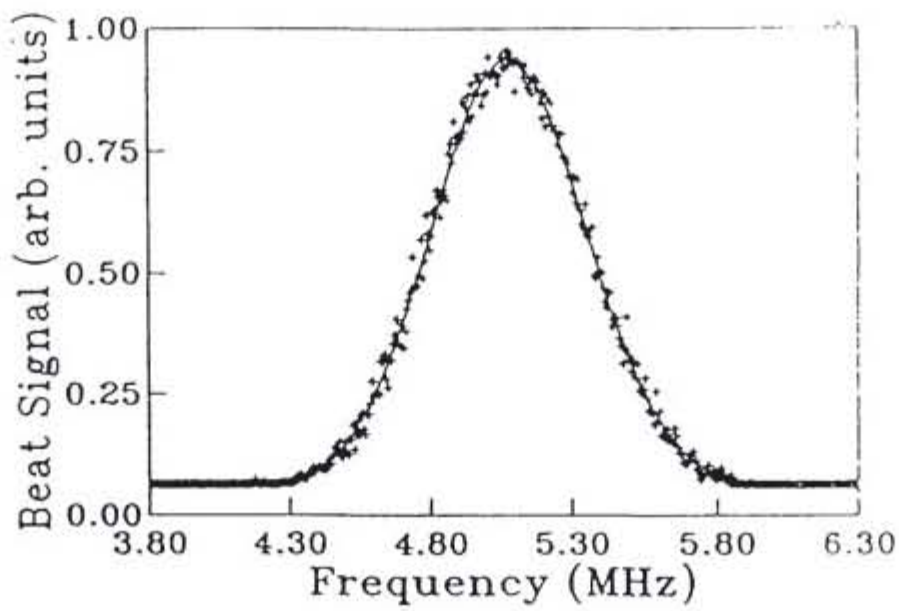

Figure 12 : Analyse spectrale du battement entre un laser à colorant commercial et un laser $\mathrm{HeNe}$ à 612 $\mathrm{nm}$. Ce profil a été modélisé par une Gaussienne de largeur à mi-hauteur de $450 \mathrm{kHz}$.

\subsubsection{Le dispositif d'asservissement du laser à colorant}

Le laser est un laser commercial Cohérent 699-21 qui peut être stabilisé sur une cavité de référence interne. Ceci a pour seul effet de supprimer les dérives du laser libre. Pour affiner la largeur de raie laser, un système d'asservissement sur une cavité Fabry-Perot de haute finesse a été développé où les corrections rapides sont appliquées à des transducteurs situés hors de la cavité laser. Il s'agit d'un modulateur acousto-optique et d'un modulateur électro-optique.

La cavité Fabry-Perot est une cavité de haute finesse dont la longueur est définie par du zérodur, de très faible coefficient de dilatation thermique. L'intervalle spectral libre est de 330 $\mathrm{MHz}$ et la largeur de raie de $250 \mathrm{kHz}$. Une modulation de phase à la fréquence de $12.5 \mathrm{MHz}$ est appliquée sur le faisceau à l'aide d'un modulateur électro-optique. Le schéma dit de PoundDrever-Hall est utilisé pour asservir la phase/fréquence du laser : on utilise comme discriminateur de fréquence le signal en réflexion à une fréquence grande devant la largeur de la cavité. La bande passante de l'asservissement (fréquence à gain unité) de $4 \mathrm{MHz}$ est elle-même, grande devant la largeur de la cavité.

Le signal réfléchi est l'interférence entre le faisceau laser réfléchi sur le miroir d'entrée et le faisceau issu de la lumière stockée et filtrée par la cavité sur un temps de l'ordre de $\tau_{c}$. Il existe alors deux régimes d'asservissement : sur des temps plus longs que $\tau_{c}$, le régime est quasistatique et le signal d'erreur est bien représenté par la Figure 14.a. On a affaire à un asservissement en fréquence. Sur des temps courts devant le temps de stockage, $\tau_{c}$, de la lumière dans la cavité, les fluctuations de phase du laser incident n'affectent nullement la lumière stockée dans la cavité : le signal d'erreur est sensible à la différence de phase entre les deux faisceaux qui ne sont pas corrélés sur cette échelle de temps. On peut montrer qu'il s'opère un asservissement en phase du laser incident sur le faisceau de référence qui caractérise la cavité. Dans ce cas, on s'aperçoit que le délai de transmission de l'information utile à 
l'asservissement n'est pas limité par le temps de stockage de la lumière dans la cavité de référence comme pour un asservissement sur le pic de transmission.

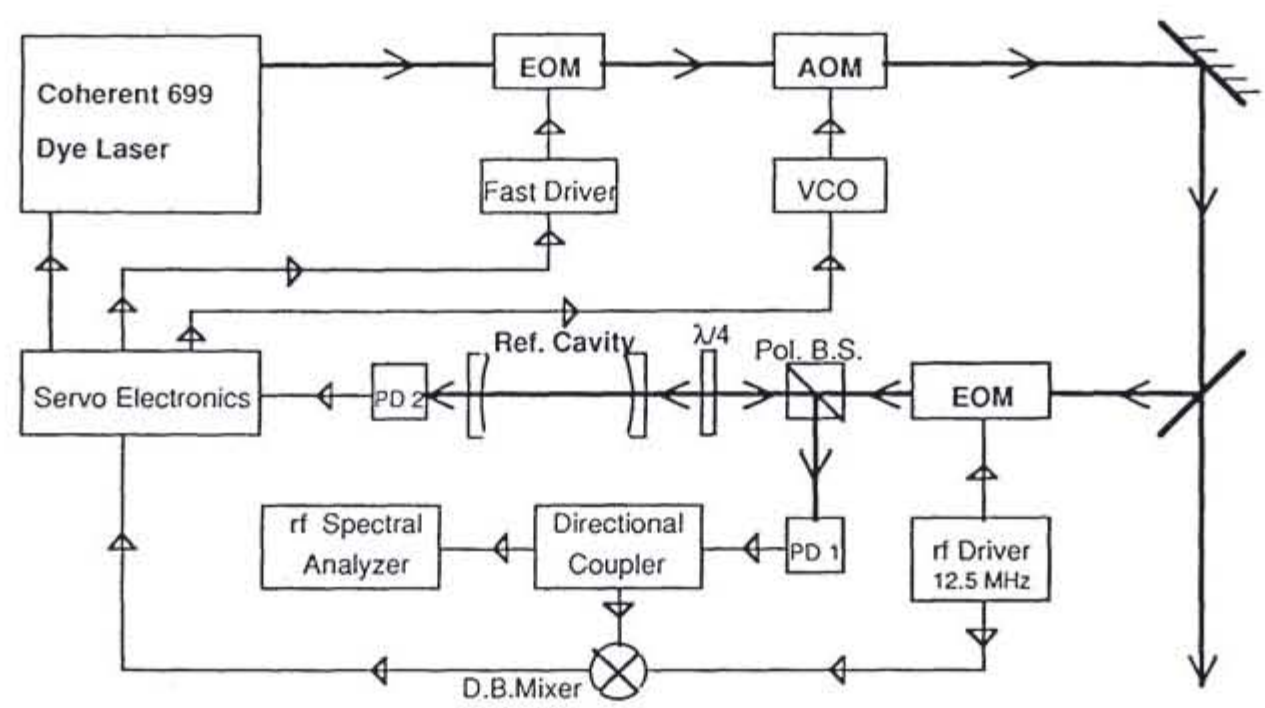

Figure 13 : Block-Diagramme de l'asservissement en fréquence du laser à colorant sur une cavité de haute finesse.
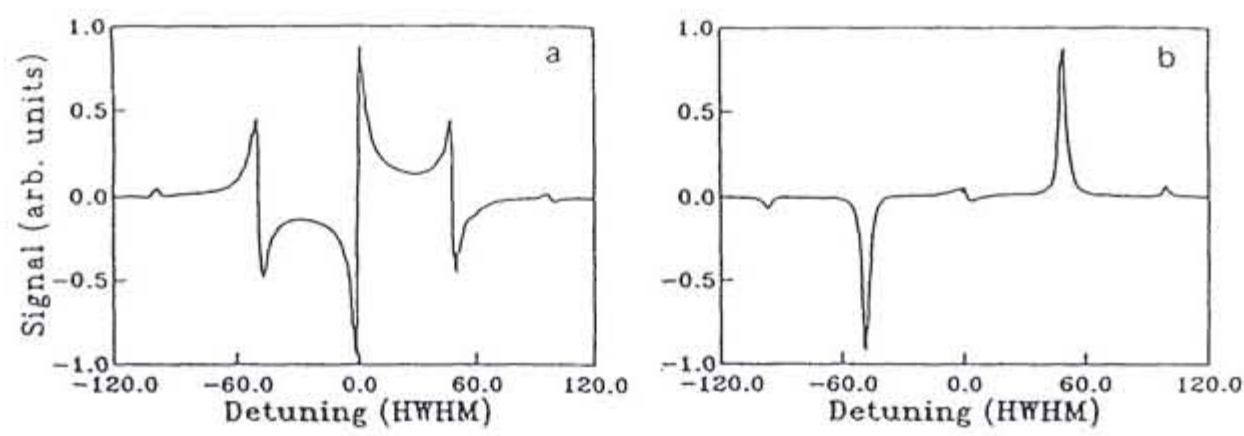

Figure 14 : signal de réflexion détecté sur la photodiode PD 1 (Fig. 13) en quadrature (a) utilisé pour l'asservissement et en phase (b). 
La correction de la fréquence laser est appliquée en plusieurs points. Le signal de transmission (PD 1) est utilisé pour stabiliser sur le long terme la longueur de la cavité laser. Le signal réfléchi (PD 2) après démodulation à $12.5 \mathrm{MHz}$ est traité et filtré (servo électronics) puis appliqué sur le modulateur acousto-optique à l'aide d'un oscillateur contrôlé en tension (VCO) et sur le modulateur électro-optique (EOM). La bande passante de la correction sur le modulateur acousto-optique est limitée à environ $100 \mathrm{kHz}$ à cause du délai de propagation de l'onde acoustique dans le cristal. La bande passante de l'ensemble de l'asservissement de 4 $\mathrm{MHz}$ est atteinte grâce à l'ultime correction appliquée sur le cristal électro-optique.

Les performances de l'asservissement sont évaluées en observant le signal d'erreur à l'analyseur de spectre vers $12.5 \mathrm{MHz}$ (Figure 15).

Pour l'interprétation des mesures, deux paramètres sont importants : la puissance associée au bruit de photons dans la bande passante de la mesure (soit $1 \mathrm{kHz}$ ), elle vaut $-83 \mathrm{dBm}$ au point de fonctionnement de l'asservissement ; la dépendance de l'excursion en fréquence par rapport à la densité spectrale de puissance mesurée également au point de fonctionnement de l'asservissement. Cette calibration montre que le bruit de photons sera interprété par l'asservissement comme un bruit blanc de fréquence de $\sqrt{f_{m s}^{2} / B}=0.395 \mathrm{~Hz} / \sqrt{\mathrm{Hz}}$. Le signal d'erreur atteint ce niveau dans le cas du gain maximum (courbe inférieure). Il descend même endessous dans la bande $0-100 \mathrm{kHz}$ : dans ce cas, le bruit de photons est transformé en fluctuations de fréquence par l'asse-vissement. Ceci donne la largeur ultime de la raie du laser asservi, soit d'après Eq. (26), $\Delta v=0.49 \mathrm{~Hz}$.

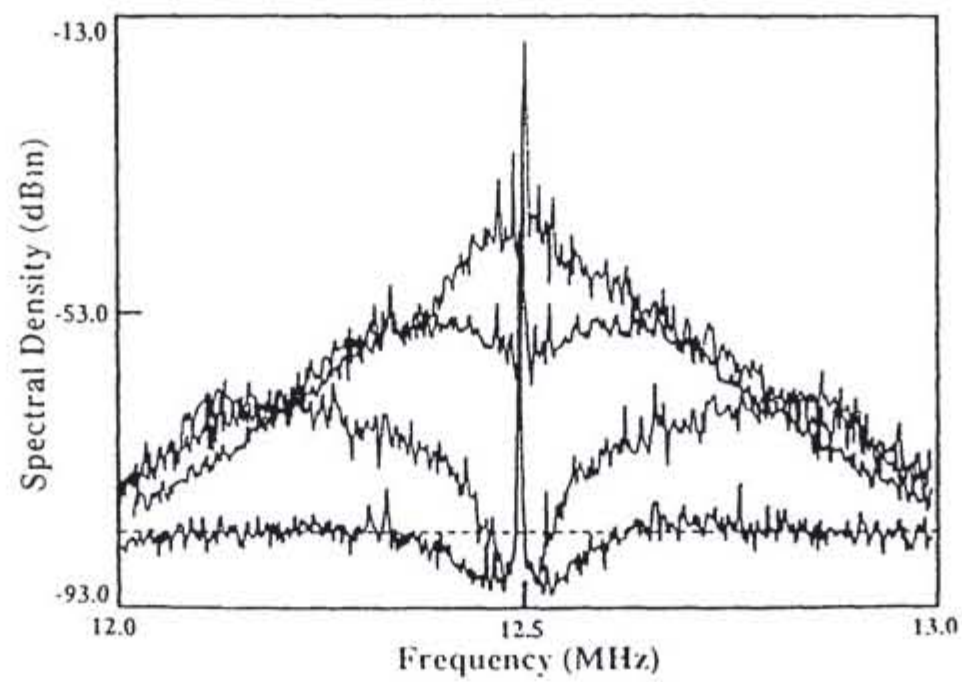

Figure 15 : Analyse spectrale du signal d'erreur. La courbe du haut représente le laser asservi seulement sur sa propre cavité. Les courbes successives sont obtenues en augmentant le gain de $10 \mathrm{~dB}$. La ligne en pointillé représente le niveau du bruit de photons $(-83 \mathrm{dBm})$ dans la bande vidéo de $1 \mathrm{kHz}$ avec laquelle ces mesures sont effectuées.

Rem \#1 : Cette limite ultime n'est atteinte qu'avec un asservissement présentant une bande passante grande devant la largeur de raie du laser libre $(450 \mathrm{kHz})$.

Rem \#2: Si la finesse de la cavité est accrue d'un facteur 10, alors la largeur de raie laser serait dans les mêmes conditions divisée par 100 d'après Eq. (50).

Rem \#3: L'asservissement assure en fait une recopie de la raie laser sur la fréquence de résonance de la cavité de référence au bruit de photons près. Les mesures ci-dessus ne montrent que l'écart entre les spectres. En particulier, tout bruit de la longueur de la cavité est transféré 
sous forme de bruit de fréquence qui ne pourra être visualisé qu'en observant le battement entre les fréquences de deux systèmes indépendants.

\subsection{Asservissement de deux lasers HeNe sur deux modes d'une même cavité Fabry-Perot (D'après [7])}

Deux lasers $\mathrm{HeNe}$ identiques sont asservis en fréquence sur deux fréquences de résonance consécutives d'une cavité Fabry-Perot de haute finesse $(\mathrm{F}=3500)$ dont l'intervalle spectral libre est environ $242.7 \mathrm{MHz}$. Fig. 16 représente le montage expérimental. Différents systèmes d'isolation optiques (modulateurs acousto-optiques, isolateurs de Faraday et lames quart d'ondes) sont insérés pour réduire très fortement les effets d'interférence parasite qui introduisent des déplacements lents du point de fonctionnement des asservissements.

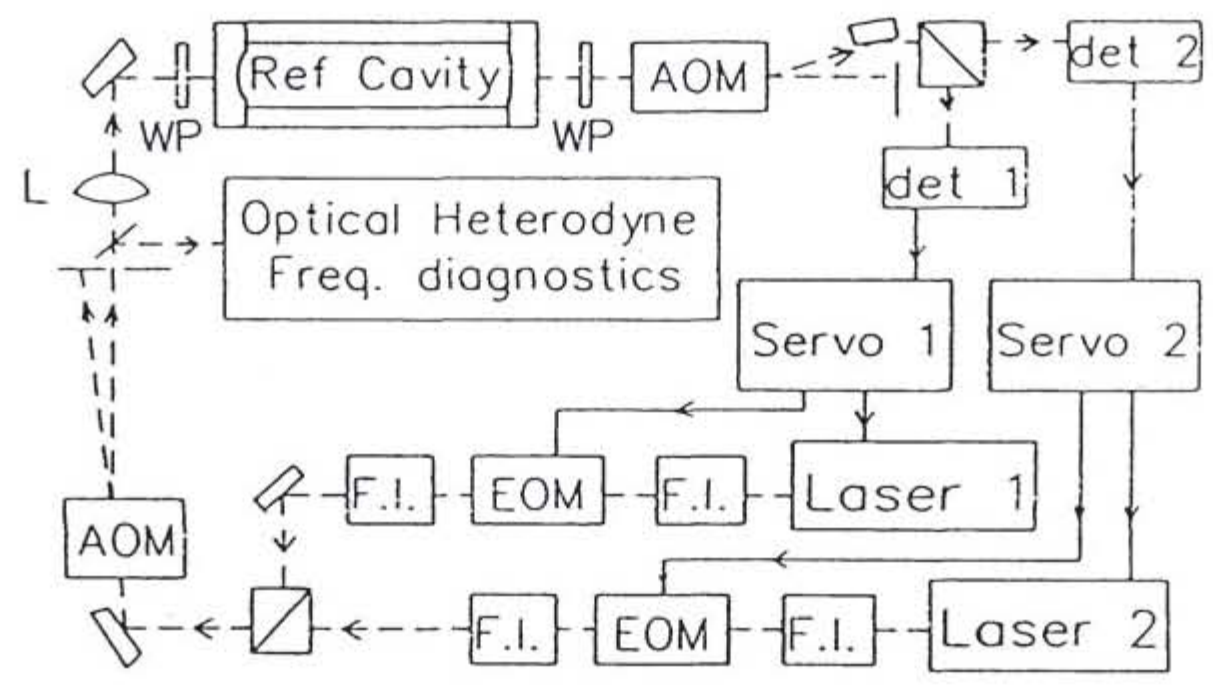

Figure 16 : Dispositif expérimental : F.I. : Isolateur de Faraday, W.P. : lame quart d'onde.

La fréquence de modulation de $25 \mathrm{kHz}$ est appliquée à l'aide d'un modulateur électrooptique. Les signaux d'erreur associés aux deux faisceaux lasers qui présentent des polarisations croisées sont détectés sur deux détecteurs (det. 1 et 2) en transmission de la cavité Fabry-Perot. La correction de fréquence est appliquée sur trois transducteurs : un cordon chauffant appliqué sur le tube laser compense les dérives lentes de la longueur de la cavité (constante de temps de quelques secondes); un élément piézo électrique, en créant des contraintes sur le tube laser, assure les corrections entre dc et $500 \mathrm{~Hz}$. Enfin, les corrections rapides sont appliquées sur le courant de décharge du laser qui module l'indice du milieu, la bande passante de cette correction peut s'étendre jusqu'à $25 \mathrm{kHz}$. La bande passante de l'asservissement est, quant à lui, de $5 \mathrm{kHz}$.

Le battement entre les deux lasers est détecté et analysé avant la cavité de référence. La Figure 17 montre le spectre du battement dont la largeur à mi-hauteur est de $50 \mathrm{mHz}$. 


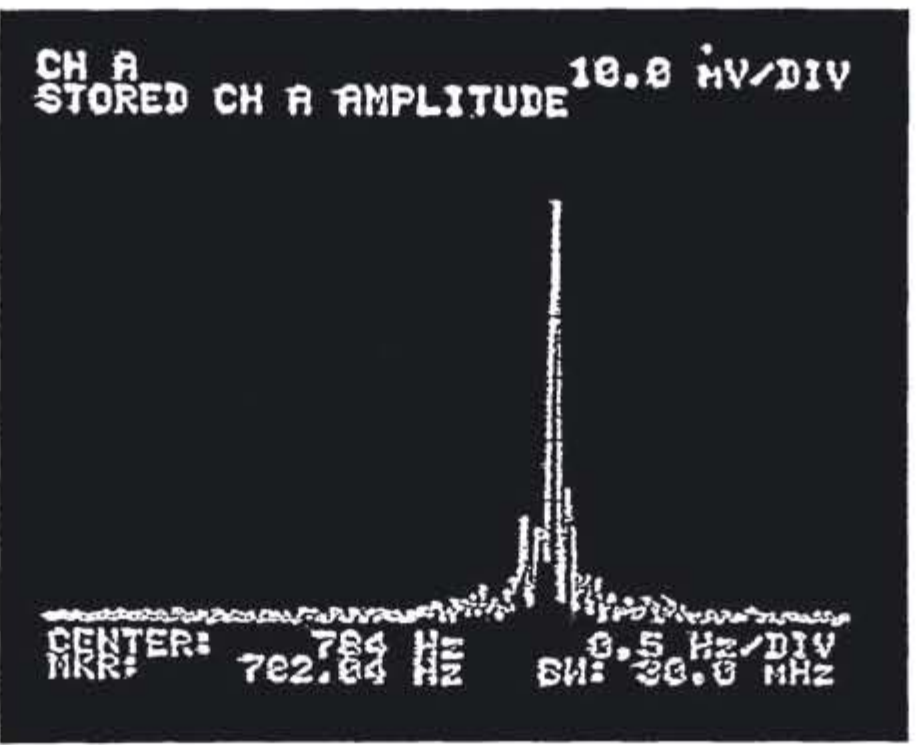

Figure 17 : Spectre de battement entre les deux lasers asservis sur deux modes adjacents de la cavité FabryPerot.

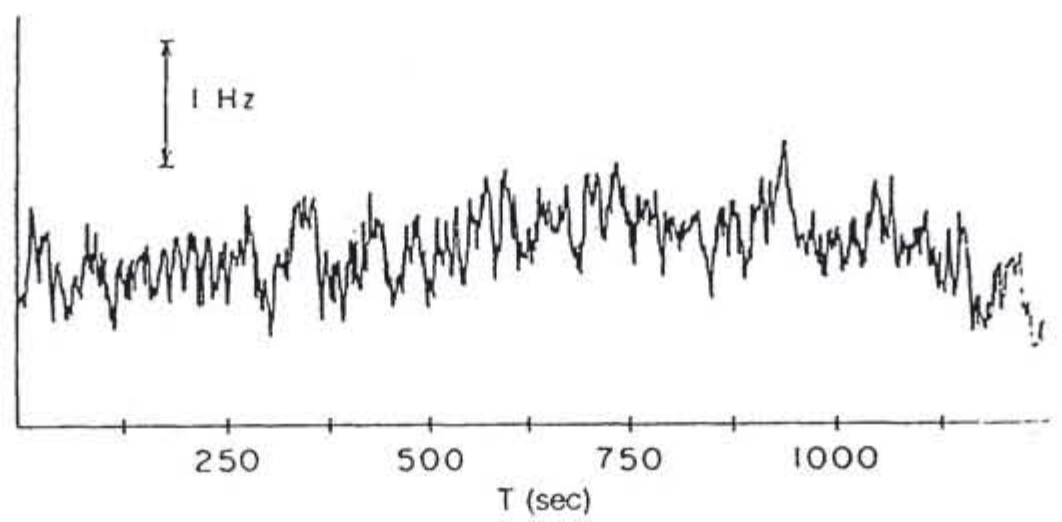

Figure 18 : Fréquence de battement enregistré sur 21 minutes 


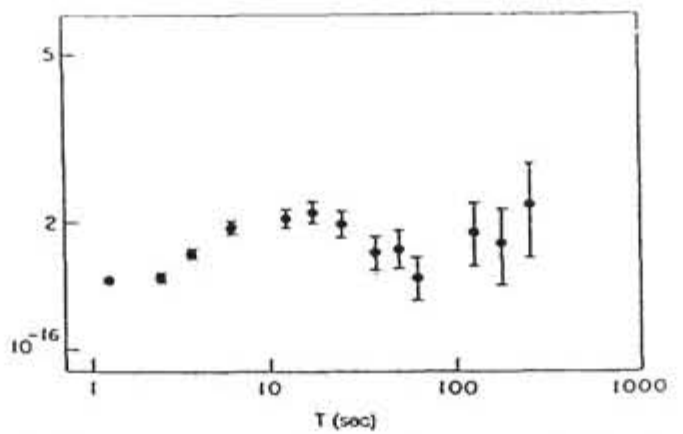

Figure 19 : Variance d'Allan du battement ci-dessus. Elle est rapportée à la fréquence optique, soit 474 $\mathrm{THz}$.

La Figure 18 montre la fréquence de battement enregistrée 1000 fois sur une vingtaine de minutes. La déviation standard de $0.29 \mathrm{~Hz}$ est à comparer avec la différence entre les extrema qui vaut $1.7 \mathrm{~Hz}$, traduisant une bonne stabilité à court terme mais la présence d'un bruit basse fréquence. Ceci est confirmé par la variance d'Allan sur la Fig. 19 qui montre que cette variance reste à niveau quasi-constant entre 1 et $300 \mathrm{~s}$. Ce plateau est caractéristique d'un bruit en I/f. Il se situe à environ $1.5 \cdot 10^{-16}$ de la fréquence optique $(474 \mathrm{THz})$ soit $70 \mathrm{mHz}$ pour chaque laser.

(a)

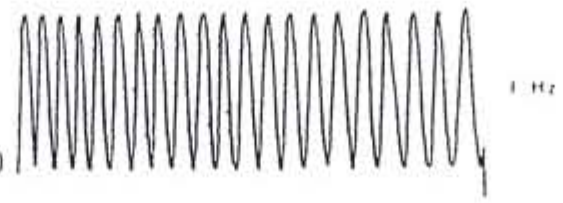

(b)

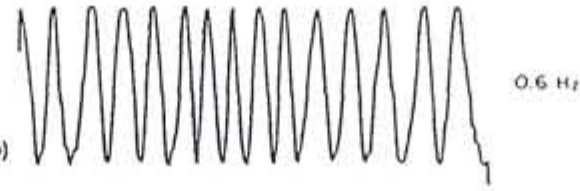

(c)
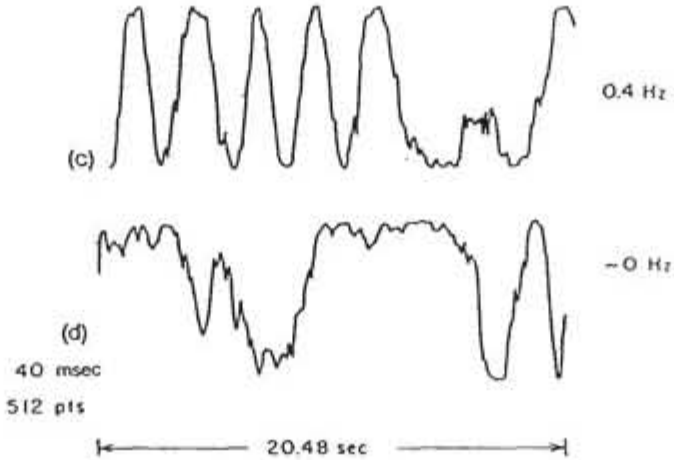

Figure 20 : Analyse temporelle de la fréquence de battement des deux lasers ramené à basse fréquence par mélange hétérodyne. Dans le cas du battement à fréquence nulle (d), il y a eu une période pendant laquelle la phase est restée constante. 
Le battement entre les deux lasers est lui-même mélangé à un signal radio-fréquence de fréquence très voisine pour générer un signal très basse fréquence qui est visualisé sur la Figure 20. On notera sur le dernier spectre (d) qu'une phase stable du signal pendant $8 \mathrm{~s}$ ne peut être obtenue qu'avec des lasers de largeur $-50 \mathrm{mHz}$.

La fréquence de battement entre les deux lasers reproduit en fait la différence de fréquence entre deux modes adjacents de la cavité, $v_{\mathrm{q}} \approx q \mathrm{qc} / 2 \mathrm{~L}$ et $\mathrm{v}_{\mathrm{q}+1} \approx(\mathrm{q}+1) \mathrm{c} / 2 \mathrm{~L}$, où $\mathrm{L}$ est la longueur optique de la cavité. De ce fait, les effets sur le battement des fluctuations de la fréquence propre de la cavité sont atténués par un facteur q $10^{6}$, ordre du mode longitudinal utilisé. Ils sont en général tout à fait négligeables dès que la cavité est bien conçue. Par exemple, une dérive de la fréquence de résonance de la cavité de $1 \mathrm{~Hz} / \mathrm{s}$ se traduira par la même dérive de la fréquence de chaque laser asservi mais elle ne se traduira sur la fréquence de battement que par une dérive de $1 \mu \mathrm{Hz} / \mathrm{s}$. C'est pourquoi cette expérience caractérise essentiellement les performances de l'asservissement lui-même et non la qualité des références de fréquence.

\section{Annexe : Le théorème de Cutler}

Il établit le lien entre propriétés spectrales d'un oscillateur et variance d'Allan. On explicite la définition de la variance d'Allan (36):

$$
\sigma_{y}^{2}(\tau)=\frac{1}{2} \lim _{T \rightarrow+\infty} \frac{1}{2 T} \int_{-\tau}^{\tau}\left(\frac{1}{\tau} \int_{t+\tau}^{t+2 \tau} y\left(t^{\prime}\right) d t^{\prime}-\frac{1}{\tau} \int_{t}^{t+\tau} y\left(t^{\prime}\right) d t^{\prime}\right)^{2} d t
$$

Utilisant la relation (33) entre $y(t)$ et $(t)$ et $(t)$, on obtient :

$$
\sigma_{y}^{2}(\tau)=\frac{1}{8 \pi^{2} v_{0}^{2} \tau^{2}} \lim _{T \rightarrow+\infty} \frac{1}{2 T} \int_{-T}^{T}(\varphi(t+2 \tau)+\varphi(t)-2 \varphi(t+\tau))^{2} d t
$$

Ce qui s'exprime à l'aide de la fonction d'autocorrélation de $\varphi$ :

$$
\sigma_{y}^{2}(\tau)=\frac{1}{4 \pi^{2} v_{0}^{2} \tau^{2}}\left(3 C_{\varphi}(0)+C_{\varphi}(2 \tau)-4 C_{\varphi}(\tau)\right)
$$

On utilise le théorème de Wiener-Khintchine (4) et les relations (12) et (39) :

$$
\sigma_{y}^{2}(\tau)=\frac{1}{8 \pi^{3} \tau^{2}} \int_{-\infty}^{+\infty} \frac{S_{y}(\Omega)}{(\Omega / 2 \pi)^{2}}(3+\exp (2 i \Omega \tau)-4 \exp (i \Omega \tau)) d \Omega
$$

Après transformation de la partie réelle qui, seule, donne une contribution non nulle, on obtient le théorème de Cutler :

$$
\sigma_{y}^{2}(\tau)=\frac{1}{\pi} \int_{-\infty}^{+\infty} S_{y}(\Omega) \frac{\sin ^{4}(\Omega \tau / 2)}{(\Omega \tau / 2)^{2}} d \Omega
$$

Note : Il faut préciser que l'on rencontre ce théorème sous une forme parfois différente à cause de la définition retenue pour la densité spectrale de puissance : utilisation des seules fréquences positives, facteur $2 \pi$ du à la définition de la transformée de Fourier. Il est donc important de vérifier la cohérence des formules utilisées.

\section{Références citées ou pour aller plus loin}

[1] Clairon A., Laurent Ph., Santarelli G., Ghezali S., Lea S. N. et Bahoura M., "A caesium fountain frequency standard : preliminary results", Proceedings de la CPEM'94 (1994). 
[2] Clairon A., Dahmani B., Filimon A. et Rutman J., IEEE Trans. on Instrum. and Measur., IM-34, (1985) 265.

[3] Clairon A., Acef O., Chardonnet Ch. et Bordé Ch. J., "State-of-the Art for High Accuracy Frequency Standards in the $28 \mathrm{THz}$ Range Using Saturated Absorption Resonances of $\mathrm{OsO}_{4}$ and $\mathrm{CO}_{2}{ }^{\prime \prime}$, in Frequency Standards and Metrology. (Ed. : A. de Marchi. Springer-Verlag Berlin, Heidelberg 1989) pp 212-221.

[4] Acef O., Zondy J. J., Abed M., Rovera D. G., Gérard A. H., Clairon A., Laurent Ph., Millerioux $\mathrm{Y}$. et Juncar P., "A $\mathrm{CO}_{2}$ to visible optical frequency synthesis chain : accurate measurement of the $473 \mathrm{THz} \mathrm{HeNe} / \mathrm{I}_{2}$ laser" Opt. Commun. 97, (1993) 29-34.

[5] Chardonnet Ch., Guernet F., Charton G. et Bordé Ch. J., "Ultrahigh-resolution Saturation Spectroscopy Using Slow Molecules in an External Cell" Appl. Phys. B 59, (1994) 333-343.

[6] Elliott D. S., Roy Rajarshi et Smith S. J., "Extracavity laser band-shape and bandwidth modification"Phys. Rev. A26, (1982) 12-18.

[7] Salomon Ch., Hils D. et Hall J. L., "Laser stabilization at the millihertz level" J. Opt. Soc. Am. B 5 (8), (1988) 1576-1587.

[8] Zhu M. et Hall J. L., "Stabilization of optical phase/frequency of a laser system : application to a commercial dye laser with an external stabilizer" J. Opt. Soc. Am. B10 (5), (1993) 802-816.

[9] Schenzle A., DeVoe R. G. et Brewer R. G., "Phase-modulation laser spectroscopy" Phys. Rev. A 25, (1982) 2606-2621.

[10] Drever R. W. P., Hall J. L., Kowalski F. V., Hough J., Ford G. M., Munley A. J. et Ward H., "Laser phase and frequency stabilization using an optical resonator" Appl. Phys. B 31, (1983) 97-105.

[11] Hils D. et Hall J. L., "Response of a Fabry-Perot cavity to phase modulated light"Rev. Sci. Instrum. 58 (8), (1987) 1406-1412.

[12] Wong N. C. et Hall J. L., "Servo-control of amplitude modulation in frequencymodulation spectroscopy : demonstration of shot-noise-limited detection" J. Opt. Soc. Am. B 2 (9), (1985) 1527-1533.

[13] Niebauer T. M., Faller J. E., Godwin H. M., Hall J. L. et Barger R. L., "Frequency stability measurements on polarization-stabilized He-Ne lasers" Appl. Optics 27 (7), (1988) $1285-1289$.

[14] Hils D., Faller J. E. et Hall J. L., "Practical sound-reducing enclosure for laboratory use" Rev. Sci. Instrum. 57 (10), (1986) 2532-2534.

[15] Hall J. L., Long-Sheng M. et Kramer G., "Principles of optical phase-locking : application to internal mirror He-Ne lasers phase-locked via fast control of the discharge current" IEEE J. Quantum Electron. 23 (4), (1987) 427-437.

[16] Henry C. H., "Theory of the linewidth of semiconductors lasers" IEEE J. Quantum Electron. 18 (2), (1982) 259-264.

[17] Laurent Ph., Clairon A. et Bréant Ch., "Frequency noise analysis of optically self-locked diode lasers" IEEE J. Quantum Electron. 25 (6), (1989) 1131-1142.

[18] Shoemaker D., Brillet A., Man C. N., Crégut O. et Kerr G., "Frequency-stabilized laserdiode pumped Nd:YAG laser" Opt. Lett. 14 (12), (1989) 609-611.

[19] Ueda K. I. et Uehara N., "Laser diode pumped solid state lasers for gravitational wave antenna", (SPIE 1837, 1992), pp. 336-345.

[20] Uehara N. et Ueda K. I., "Frequency stabilization of two diode-pumped Nd:YAG lasers locked to two Fabry-Perot Cavities" Jpn. J. Appl. Phys. 33, (1994) 1628-1633.

[21] Rovera G. D., Santarelli G. et Clairon A., "A laser diode system stabilized on the caesium $\mathrm{D}_{2}$ line". Sci. Instrum. 65 (5), (1986) 1502-1505. 